\author{
Cite this: Chem. Soc. Rev., 2012,41,6745-6759
}

www.rsc.org/csr

TUTORIAL REVIEW

\section{Dislocations in icosahedral quasicrystals $\dagger$}

\author{
Michael Feuerbacher* \\ Received 20th April 2012 \\ DOI: $10.1039 / \cos 35150 a$
}

Dislocations in quasicrystals, as a direct result of the lack of translational symmetry in these materials, possess various salient features. The Burgers vector of a dislocation in an icosahedral quasicrystal is a 6-dimensional vector, which reflects the fact that the dislocation, besides the phonon-type strain field analogous to dislocations in ordinary crystals, is associated inseparably with a further type of defect, the phasons. Phasons are critically involved in the formation and motion of dislocations in quasicrystals and govern the macroscopic plastic behaviour of these materials. In this article the properties of dislocations in icosahedral quasicrystals are comprehensively reviewed, starting from a continuum-mechanical description, via core-structure simulation, to their full experimental characterization. The experimental results presented address the icosahedral phases in the well explored systems Al-Pd-Mn and $\mathrm{Zn}-\mathrm{Mg}-\mathrm{Dy}$.

\section{Introduction}

Quasicrystals (QCs) are materials possessing long range order but no translational symmetry. Their structures have symmetry axes that were, at the time, "forbidden" by crystallographic dogmata dating back to ancient studies e.g. by Kepler. ${ }^{1}$ It was generally believed that crystal structures including rotational axes of fivefold and higher than sixfold symmetry cannot exist. The first quasicrystalline phase was discovered by Shechtman in 1982

Institut für Mikrostrukturforschung, Forschungszentrum Jülich GmbH, 52425 Jülich, Germany. E-mail: m.feuerbacher@fz-juelich.de

$\dagger$ Part of a themed issue on Quasicrystals in honour of the 2011 Nobel Prize in Chemistry winner, Professor Dan Shechtman.

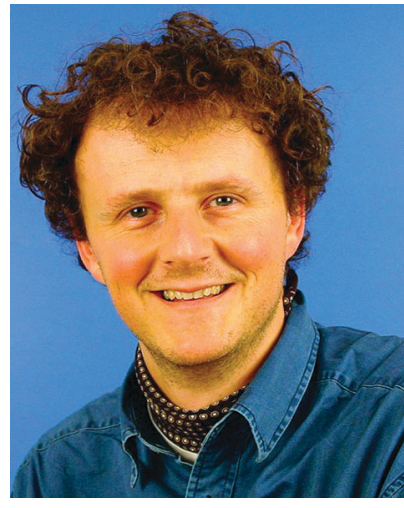

Michael Feuerbacher
Michael Feuerbacher is a research scientist at the Peter Grünberg Institute of the Forschungszentrum Jülich, Germany. Starting his professional career in the field of infrared spectroscopy of solid surfaces and thin films he moved to metal physics in 1994, specializing on Quasicrystal research. Besides the growth of large singlequasicrystals, he worked on mechanical spectroscopy, plasticity, and defect characterization. Feuerbacher was significantly involved in the development of the new field of Complex Metallic Alloys. His current projects focus on the correlation between the microscopic and macroscopic aspects of the plasticity of Complex Metallic Alloys and Quasicrystals. and the first record percepted by the scientific community appeared in $1984 .^{2}$ It reported on a metastable phase with an icosahedral-symmetric structure in a rapidly solidified $\mathrm{Al}-\mathrm{Mn}$ alloy. The icosahedral symmetry group contains fivefold axes, so the discovery was in direct conflict with the prevailing opinion. Accordingly, Shechtman's discovery led to an avalanche of scientific activity and triggered the development of QCs as a new field in materials science.

Today a variety of quasicrystalline materials in several alloy systems is known. Highly perfect and stable icosahedral QCs exist e.g. in the systems $\mathrm{Al}-\mathrm{Cu}-\mathrm{Fe}, \mathrm{Al}-\mathrm{Pd}-\mathrm{Mn}$ and $\mathrm{Zn}-\mathrm{Mg}-\mathrm{RE}$ (where RE stands for different rare-earth elements). Fig. 1a is a high-resolution transmission electron (TEM) micrograph of icosahedral $\mathrm{Zn}-\mathrm{Mg}-\mathrm{Dy}$ imaged along a fivefold direction. Clearly, the overall fivefold symmetry and local pentagonal features on various length scales are seen. The structure can be regarded as being organized in the form of five sets of lattice planes, mutually rotated by angles of 36 degrees. Within a set of lattice planes two basic interplanar distances, a long and a short one, related by the irrational number of the golden mean $\tau \approx 1.61803 \cdots$ are seen. Fig. $1 \mathrm{~b}$ is a corresponding electron diffraction pattern. It has discrete and sharp reflections, which clearly demonstrate the presence of long range order, and it has tenfold symmetry, corresponding to the fivefold symmetry of the direct-space structure. $\ddagger$ The icosahedral structure includes six fivefold, fifteen twofold and ten threefold axes, which are arranged as in a regular icosahedron. Fig. 1c is a corresponding stereographic projection of the icosahedral group along a twofold axis. The symbols represent the rotational symmetry of the corresponding axes. Besides the icosahedral phases, octagonal,

$\ddagger$ Electron diffraction patterns along zone axes of odd rotational symmetry have twice the symmetry of the corresponding real-space structure according to Friedel's law. 

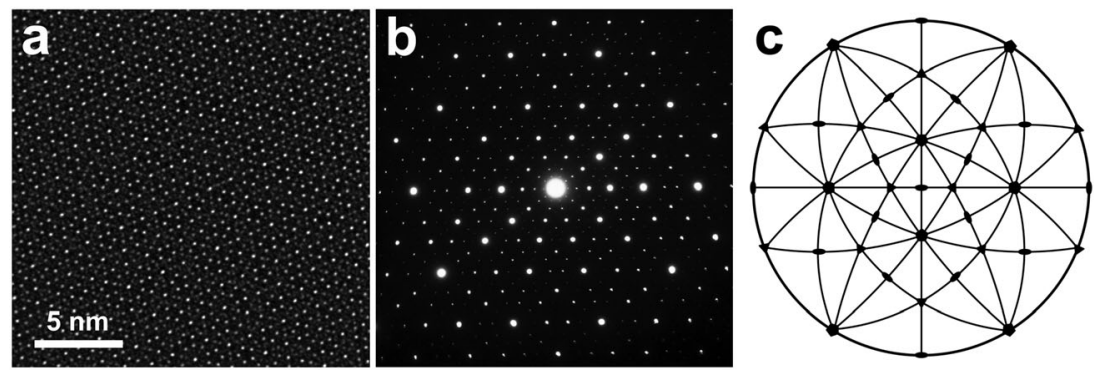

Fig. 1 High-resolution transmission electron micrograph of icosahedral $\mathrm{Zn}-\mathrm{Mg}$-Dy imaged along a fivefold direction (a) and the corresponding electron diffraction pattern (b). Stereographic projection of the icosahedral group along a twofold axis (c).

decagonal, and dodecagonal QCs, including eightfold, tenfold, and twelvefold rotational axes, respectively, were discovered to date.

Dislocations in QCs were firstly discussed in a treatment of the hydrodynamics of QCs by Levine et al. ${ }^{3}$ as early as 1985, only a few months after the report of QC discovery. The first experimental observation of dislocations was reported by Hiraga and Hirabayashi ${ }^{4}$ in 1987 . By means of high resolution TEM, these authors demonstrated the existence of dislocations in an $\mathrm{Al}-\mathrm{Mn}-\mathrm{Si} \mathrm{QC}$ and analyzed the in-plane Burgers vector by circuiting the core. Further early experimental work was carried out by Wang et al. ${ }^{5}$ on icosahedral Al-Mn QCs and by Zhang and $\mathrm{Urban}^{6}$ on decagonal $\mathrm{Al}-\mathrm{Cu}-\mathrm{Co}$.

The subject of this paper is a review of dislocations in QCs. After an introduction of the basic concepts of defects in QCs (Section 2), we will discuss dislocation structure simulations (Section 3) and dislocation analysis by means of TEM (Section 4). Experimental results for icosahedral Al-Pd-Mn will be presented and discussed, for which a respectable body of work is present in the literature (Section 5). Phasons, which are pivotally related to dislocations in QCs, will be discussed in reciprocal and real space (Section 6). In Section 7, finally, we will discuss dislocations in $\mathrm{Zn}-\mathrm{Mg}-\mathrm{Dy}$, another well investigated icosahedral structure.

Another group of QCs that has been extensively investigated with respect to the plastic properties and dislocation structures are the decagonal phases in the system Al-Ni-Co. Decagonal phases possess a highly anisotropic structure that can be understood as a periodic stacking of quasiperiodic planes, which leads to a distinct anisotropy of the plastic properties and the dislocation microstructure. The existence range of decagonal $\mathrm{Al}-\mathrm{Ni}-\mathrm{Co}$ extends over a wide range of compositions, which is also reflected in the plastic properties. As a result, the plasticity of decagonal structures is highly complex and requires a careful distinction of cases regarding deformation geometry and sample composition. This would exceed the scope of the present paper and hence we limit this review to dislocations in icosahedral structures and for the decagonal case refer to existing comprehensive treatments in the literature. ${ }^{7,8}$

\section{Defects in quasicrystals}

Defects in QCs were firstly discussed by Levine et al. ${ }^{3}$ The authors treated the hydrodynamics of 2- and 3-dimensional icosahedral lattices in a density wave-approach and identified dislocations as stable structural defects in QCs. Indexing of a dislocation Burgers vector, unlike the case in ordinary crystals, was found to require more indices than real-space dimensions. The number of indices corresponds to the number of independent hydrodynamic variables, which in turn corresponds to the number of relatively incommensurate lengths. The authors write: "The Burgers vector lattice is a $2 \times d$ dimensional lattice which is distinct from the $d$-dimensional real-space lattice of atomic positions". The Burgers vector lattice represents the entirety of vectors containing the possible Burgers vector in a given structure. In the icosahedral case, $d=3$, and hence the Burgers vector is 6-dimensional.

Socolar et al..$^{9}$ demonstrated that the hydrodynamic degrees of freedom in a QC can be parameterized by two vectors, $\boldsymbol{u}_{\|}$and $\boldsymbol{u}_{\perp}$. In full analogy to the case of periodic crystals, uniform shifts in $\boldsymbol{u}_{\|}$correspond to a translation of the system and spatial variations in $\boldsymbol{u}_{\|}$give rise to propagating phonons. Statically this corresponds to the occurrence of continuous deviations from the ideal atomic positions, for example smooth curvatures in atomic rows. We will refer to this as phonon-type displacement. Variations in $\boldsymbol{u}_{\perp}$, on the other hand, lead to discrete alterations of the atomic positions, which in high resolution electron micrographs are seen as jags or shifts in atomic rows. Mathematically the variable $\boldsymbol{u}_{\perp}$ is analogous to the phason degree of freedom in incommensurate crystals and is therefore referred to as phason displacement.

A dislocation in a $\mathrm{QC}$ is associated with spatial variations both in $\boldsymbol{u}_{\|}$and $\boldsymbol{u}_{\perp}$ and its Burgers vector is a net measure of these displacements about any circuit enclosing the dislocation core. We define two components of the Burgers vector,

$$
\boldsymbol{b}_{\|}=\oint_{c} \mathrm{~d} \boldsymbol{u}_{\|} \text {and } \boldsymbol{b}_{\perp}=\oint_{c} \mathrm{~d} \boldsymbol{u}_{\perp},
$$

where the integration is carried out on a closed path around the dislocation core. The parallel component $\boldsymbol{b}_{\|}$describes the continuous phonon-type displacements associated with the dislocation, and is therefore also referred to as the phonon component. The perpendicular component $\boldsymbol{b}_{\perp}$ is specific to QCs and describes the phason strain associated with the dislocation. It is therefore also referred to as the phason component. In icosahedral QCs, both $\boldsymbol{b}_{\|}$and $\boldsymbol{b}_{\perp}$ are 3-dimensional vectors. Each Burgers vector necessarily corresponds to nonzero $\boldsymbol{u}_{\|}$and $\boldsymbol{u}_{\perp}$, so that dislocations in QCs, unlike those in periodic crystals, cannot be interpreted in terms of insertions or removals of halfplanes in physical space. 
A density wave approach is highly powerful for the general discussion of elasticity and basic defect structures in QCs. If it comes to the description of the atomic structures of QC materials, however, the so-called higher dimensional crystallography ${ }^{10,11}$ has gained the highest practical relevance. In these terms, the real-space atom distribution is constructed on the basis of a periodic higher-dimensional hyperlattice. Since the hyperlattice is periodic it can be represented in closed form by a higher-dimensional unit cell.

The higher-dimensional unit cell, which defines a hypercrystal coordinate system, is, analogous to the case of ordinary crystals, translated in the higher-dimensional space to form a hyperlattice. For the construction of the QC lattice in physical space a second, orthonormal coordinate system is introduced, which is rotated with respect to the first. The second system is subdivided into two subspaces. The first subspace represents the real, physical space. It is also referred to as parallel space and contains vectors $\boldsymbol{x}_{\|}$. The second subspace, spanned by the remaining hyperspace directions, is referred to as perpendicular space and contains vectors $\boldsymbol{x}_{\perp}$. The geometry is visualized in Fig. $2 \mathrm{a}$ for the case of a 1-dimensional QC, which is constructed on the basis of a 2-dimensional hyperlattice. The edges of the higher-dimensional unit cell (dotted) define the hypercrystal coordinate system. The second coordinate system (grey) is rotated with respect to the first and spans the subspaces $\boldsymbol{E}_{\|}$and $\boldsymbol{E}_{\perp}$. The rotation angle between the two coordinate systems corresponds to the number of the golden mean

$$
\tau=\frac{\sqrt{5}+1}{2}
$$

Since $\tau$ is an irrational number, besides the origin no further hyperlattice point falls into the subspace $\boldsymbol{E}_{\|}$.

For the construction of icosahedral QCs, the hyperlattice is 6-dimensional and physical and perpendicular space each is 3-dimensional. The relative orientation of the coordinate systems is chosen such that the projections of the six axes of the hypercrystal system are oriented along the six fivefold axes
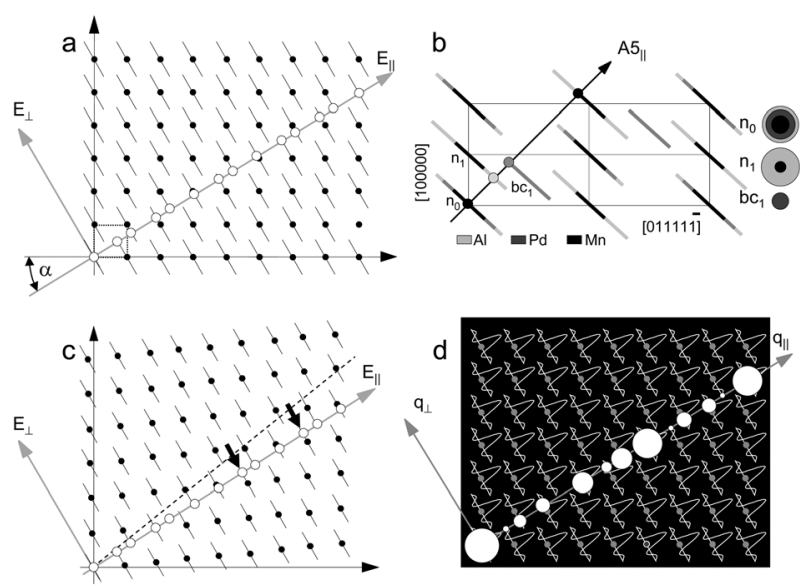

Fig. 2 Construction of an ideal 1-dimensional QC by the intersection procedure (a) and hyperlattice unit cell employed in the structure model of icosahedral Al-Pd-Mn (b). Construction of a 1-dimensional QC in the presence of linear phason strain (c) and construction of the diffraction pattern (d) corresponding to the ideal QC in (a). of an icosahedron. This ensures that the structure in physical space has icosahedral symmetry. It also has the consequence that the rotation matrix describing the relation between the two coordinate systems has irrational entries involving the golden mean $\tau .^{12}$

The higher-dimensional unit cell is decorated by a basis of atomic hypersurfaces. These are 3-dimensional objects that have only extensions in perpendicular space. The atomic positions of the QC are determined by the intersection points of the atomic hypersurfaces with physical space $\boldsymbol{E}_{\|}$. In the 1-dimensional case of Fig. 2a, the hypersurfaces are lines and the atom positions (open circles), defined by the intersection points with physical space, form a linear quasiperiodic arrangement. The atom positions are separated by long and short distances, which are nonperiodically but deterministically arranged throughout the construction. The length ratio of the long and short distances is determined by the rotation angle $\alpha=\arctan \left(\tau^{-1}\right)$ between the two coordinate systems and equals $\tau$.

This construction method, which is referred to as the "intersection procedure", can by adequate definition of the hypersurfaces also specify the site occupation by different atomic species. An example is shown in Fig. 2b. For the construction of the atomic structure of icosahedral A1-Pd- $\mathrm{Mn}^{13,14}$ a face centred 6-dimensional unit cell decorated with 3-dimensional hypersurfaces is used. On the three hyperlattice positions $\mathrm{n}_{0}, \mathrm{n}_{1}$, and $\mathrm{bc}_{1}$, three different hypersurfaces are placed, two of which consist of concentric spheres assigned to different elements. The figure shows the plane spanned by the hyperlattice directions [100000] and [011111] , which contains the fivefold physical-space direction $\mathrm{A} 5{ }_{\|}$. The intersections of the hypersurfaces with $\mathrm{A} 5$ ॥ determine the physicalspace atomic positions along that specific direction, and the radius at which the hypersurfaces are intersected determines the individual atomic species. In this structure model, spherical hypersurfaces are used, which is a very convenient approximation allowing for fast calculation of the atom positions. However, such "spherical models" have the drawback that occasionally unphysical short distances occur in physical space. More elaborated models, as constructed for icosahedral Al-Pd-Fe, ${ }^{15}$ employ icosahedral symmetric hypersurfaces (e.g. truncated triacontahedra) and avoid such artefacts.

Note that the arrangement of atom sites in physical space constructed by the intersection procedure depends on the choice of origin. Physical space can be translated along perpendicular space, which will lead to different but similar atomic arrangements in the QC. The resulting QCs can be subdivided into different local isomorphism classes. Two QCs are in the same local isomorphism class (LI class) if every finite atom arrangement in each is found in the other. If two QCs are in the same LI class, this means that they cannot be distinguished by measurements on any finite length scale. Therefore they are physically indistinguishable, have the same diffraction properties and, in particular, have the same free energy. ${ }^{9}$

The hyperlattice is more than a mathematical construction procedure for ideal QC structures - it also defines displacements, defects and strain fields in QCs. In the hydrodynamic description we have introduced two displacements, $\boldsymbol{u}_{\|}$and $\boldsymbol{u}_{\perp}$. These can, in a hyperlattice approach, be interpreted as 
components of a higher-dimensional displacement field $\boldsymbol{U}$, where the component $\boldsymbol{u}_{\|}$describes the displacement field in parallel space, and $\boldsymbol{u}_{\perp}$ describes the displacement field in perpendicular space. The presence of hyperlattice displacements translates, via the intersection procedure, to displacements in the real-space structure of the QC. Displacements $\boldsymbol{u}_{\|}$obviously directly describe continuous displacements in the QC structure, which can take arbitrarily small values. Displacements $\boldsymbol{u}_{\perp}$, on the other hand, have only components along perpendicularspace directions, in which the atomic hypersurfaces are also extended. This means that some of the hypersurfaces, which in the ideal lattice intersect physical space, in the presence of $\boldsymbol{u}_{\perp}$ do not intersect anymore. As a result atom positions disappear in the QC. On the other hand, hyperlattice points situated such that their appertaining hypersurfaces in the ideal lattice did not intersect physical space, may do so in the presence of $\boldsymbol{u}_{\perp}$, which leads to the appearance of new atomic positions in the QC. By construction this can lead only to the appearance of atoms on clearly defined positions, and hence the resulting physical-space displacements are discrete. Fig. 2c exemplifies this for a strained 2-dimensional hyperlattice. The hyperlattice positions are displaced along the $\boldsymbol{E}_{\perp}$ direction, and the strength of the displacement linearly increases along $\boldsymbol{E}_{\perp}$ (dashed line). This type of hyperlattice displacement field is referred to as linear phason strain. As a result, the same atomic distances as in the ideal case occur in physical space, but at some positions their order has changed, and hence some atoms occupy different positions (arrows, $c f$. Fig. 2a). These changes can be seen as small discrete atomic jumps, which are referred to as phason jumps.

The closeness condition ${ }^{16}$ ensures that the atomic hypersurfaces are constructed such that under any displacement $\boldsymbol{u}_{\perp}$, the number of disappearing and appearing atoms is equal, that is, the number of atoms in the QC is conserved. A further condition demands that a locally varying hyperlattice strain field only depends on the physical-space coordinate $\boldsymbol{x}_{\|}$. If it were dependent on $\boldsymbol{x}_{\perp}$, the atomic arrangement in the resulting QC would depend on the placement of the hypercrystal origin along perpendicular space, which has to be considered unphysical. Hence we write

$$
\boldsymbol{U}=\boldsymbol{u}_{\|}\left(x_{\|}\right)+\boldsymbol{u}_{\perp}\left(x_{\|}\right) \text {. }
$$

Dislocations can be introduced into the QC by introducing a hyperdislocation into the hyperlattice by means of a generalized Volterra process. ${ }^{17-19}$ Since the hyperlattice is periodic, the Volterra process can be understood as insertion or removal of a hyper-halfplane. In the icosahedral case this halfplane is a 5-dimensional object, terminated by a 4-dimensional hyperdislocation line. The Burgers vector $\boldsymbol{B}$ of the hyperdislocation is 6-dimensional and can be decomposed into the components $\boldsymbol{b}_{\|}$and $\boldsymbol{b}_{\perp}$ as

$$
\boldsymbol{B}=\boldsymbol{b}_{\|}+\boldsymbol{b}_{\perp},
$$

which are, as described above, referred to as phonon and phason component of the Burgers vector respectively. By construction $\boldsymbol{B}$ is a hyperlattice translation vector, and as a result the phonon and phason components both are always nonzero. The strain accommodation parameter (SAC)

$$
\zeta=\frac{\left|\boldsymbol{b}_{\perp}\right|}{\left|\boldsymbol{b}_{\|}\right|}
$$

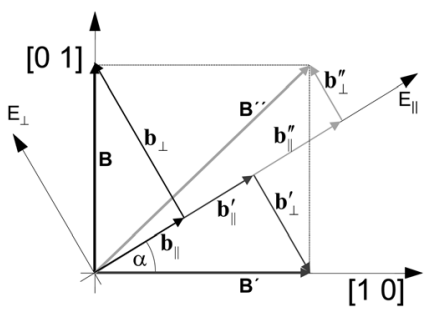

Fig. 3 Burgers vectors of different direction in the hyperlattice can lead to parallel physical-space Burgers vectors of different length and different SAC. Schematic representation for the case of a 2-dimensional hyperlattice (see text).

was introduced ${ }^{20}$ in order to specify how the strain of a given dislocation is distributed into phonon and phason parts.

Burgers vectors of different direction in the hyperlattice can lead to parallel physical-space Burgers vectors of different length and different SAC. This is schematically shown for a 2-dimensional hyperlattice, which is represented by a single hyperspace unit cell in Fig. 3 ( $c f$. dotted box in Fig. 2a). If the Burgers vector $\boldsymbol{B}$ (black) corresponds to the lattice vector along [0 1], its phason and phonon components, $\boldsymbol{b}_{\perp}$ and $\boldsymbol{b}_{\|}$, correspond to the projections on $\boldsymbol{E}_{\perp}$ and $\boldsymbol{E}_{\perp}$, respectively, and the SAC is $\zeta=\tau$. The length of $\boldsymbol{b}_{\|}$is related to the $b$-hyperlattice constant as $\boldsymbol{b}_{\|}=b \sin \alpha$. Now consider Burgers vector $\boldsymbol{B}^{\prime}$ (dark grey) corresponding to the lattice vector along [1 0]. The SAC is now $\zeta=\tau^{-1}$ and the length of $\boldsymbol{b}_{\|}^{\prime}$ is related to the $a$-hyperlattice constant as $\boldsymbol{b}_{\|}^{\prime}=a \cos \alpha$. Assuming, without loss of generality, a square hyperlattice, i.e. $a=b$, then $\left|\boldsymbol{b}_{\|}^{\prime}\right| /\left|\boldsymbol{b}_{\|}\right|=\tau$. The phonon component of the second Burgers vector is thus parallel and longer by a factor of $\tau$ than that of the first. Analogously we find that for Burgers vector $\boldsymbol{B}^{\prime \prime}$ (light grey), which forms the diagonal of the hyperlattice unit cell, the phonon component is again longer by a factor of $\tau$, and the SAC is $\zeta=\tau^{-3}$. Burgers vectors in hyperspace along different hyperlattice directions thus lead to a series of parallel Burgers vectors in physical space. The phonon components of the Burgers vectors in the series are related by factors of $\tau$ and are opposed to the corresponding series of phason components. The SAC is given by a sequence $\tau^{n}$, where $n$ are odd numbers, and for subsequent members of the series $n$ differs by 2 .

\section{Dislocation simulation}

The first simulations of dislocations were carried out by Levine et $\mathrm{al}^{3}{ }^{3}$ and later on by Socolar $e t$ al. ${ }^{9}$ by adding a suitable phase in a density-wave simulation. In these works, dislocations are identified as stable topological line defects (point defects in the 2-dimensional density wave simulations) if and only if they possess nonzero components both in $\boldsymbol{u}_{\|}$and $\boldsymbol{u}_{\perp}$. If a dislocation has only components in $\boldsymbol{u}_{\|}$the atomic arrangement at a large distance from the core cannot approach the LI class of the undefected $\mathrm{QC}$ due to the atom arrangements at the beginning and the end of a large Burgers circuit. They would only match with an arrangement of the same LI class unless $\boldsymbol{u}_{\|}$ would correspond to an exact translation vector which, however, does not exist in a quasiperiodic structure. To approach the LI class of the undefected QC at large distances from the core, as demanded for a true line defect, atom rearrangements 
corresponding to a nonzero component $\boldsymbol{u}_{\perp}$ are required. Accordingly, in the density wave images it is seen that the dislocation is a localized defect which includes curvature at the core as well as the presence of jags.

Bohsung and Trebin constructed a dislocation in a Penrose lattice. ${ }^{17}$ They demonstrated that a Volterra process in physical space does not lead to a true dislocation but to a line of vertices along the cut, not belonging to the LI-class of the initial Penrose lattice. In order to construct a true dislocation, a generalized Volterra process in hyperspace, in the case of the Penrose lattice a 5-dimensional space, is carried out: a 4-dimensional hyperplane is removed, the remaining lips are connected and the core area is relaxed by an isotropic strain field. The corresponding Penrose lattice in physical space then contains a localized dislocation. It is associated with phasons surrounding the core, and the Burgers vector is 5-dimensional. A similar construction of a dislocation in a Penrose lattice was later carried out by Edagawa et al. ${ }^{21}$

Dislocations in icosahedral Al-Pd-Mn, constructed by Yang et al. ${ }^{19}$ via a generalized Volterra process are shown in Fig. 4. The simulation employs the structure model of $\mathrm{Al}-\mathrm{Pd}-\mathrm{Mn},{ }^{14}$ which was solved on the basis of experimental X-ray and neutron scattering data by higher-dimensional crystallography (see Fig. 2d). Elastic displacement fields taking into account the symmetry of the icosahedral structure, ${ }^{22,23}$ solved for phason and phonon components were used. Therefore the dislocation simulation can be considered to be a realistic model directly comparable with experimental high-resolution TEM images.

The simulation is carried out by a generalized Volterra process in 6-dimensional hyperspace and subsequent application of the intersection procedure. A 4-dimensional hyperline $\mathrm{L}$, which is the direct sum of the desired line direction in physical space and the perpendicular space ${ }^{24}$ is chosen, and a 6 dimensional body, defined by $\mathrm{L}$ and two hyperlips is removed. The distance between the hyperlips is given by the 6-dimensional Burgers vector, which is chosen as a hyperlattice translation vector. Therefore, upon closure of the gap, the hyperlips match perfectly. The gap is closed by applying the specific calculated strain fields.

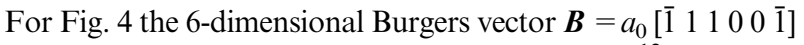
was chosen (indexing according to Cahn et al., ${ }^{12} a_{0}$ is the lattice parameter of the $6 \mathrm{D}$ hyperlattice). This is one of the experimentally observed Burgers vectors (see Section 5) and is parallel to a twofold physical-space direction. The line direction was chosen along a fivefold physical-space direction and placed through the origin of the physical-space coordinate system. The so-constructed hyperspace dislocation is then transferred to physical space by the intersection procedure. The result is shown in Fig. 4a.

The dislocation is a true 1-dimensional line defect. The core is located in the centre of the figure, and at a distance from the core the structure is essentially indistinguishable from the ideal structure. At the core, the lattice planes are curved as a result of the phason component, and around the dislocation we find jags in lattice planes, which is a result of the phonon component of the strain field. These features can be seen best in a grazing view, e.g. in the perspective representation Fig. 4c, along the direction of the arrow. The physical space component of the Burgers vector is $a^{\prime}\left[\begin{array}{lll}\overline{1} / 1 & 2 / \overline{1} & 1 / 0\end{array}\right]$ and has a length of $0.296 \mathrm{~nm}$ corresponding to a SAC of $\tau^{3}$, where $a^{\prime}=a_{0} / \sqrt{2(2+\tau)}$ and $a_{0}=0.645 \mathrm{~nm}$.

Neglecting the phason component of the Burgers vector, i.e. setting $\boldsymbol{B}=\boldsymbol{b}_{\|}$leads to the dislocation shown in Fig. $4 \mathrm{~b}$. This procedure, which corresponds exactly to the direct performance of a Volterra process in physical space, results in a dislocation terminating a planar fault, i.e. not in a true line defect. Lattice-plane curvature is present at the dislocation core, but there are no jags. This can be seen most easily in the perspective representation of Fig. 4d. In a later part of this paper we will come across an experimental situation that corresponds to this defect (Section 6).

Dislocation simulations on the basis of a realistic structure model were also carried out by Gratias et al. ${ }^{25}$ These authors used a more elaborated polyhedral structure model of icosahedral AlPd-Mn, carried out a generalized Volterra process in hyperspace, and used an isotropic strain field for relaxation of the hyperlips.

\section{Burgers vector analysis in the TEM}

Imaging and analysis of dislocations in QCs can be carried out by Bragg-contrast imaging or high-resolution TEM.
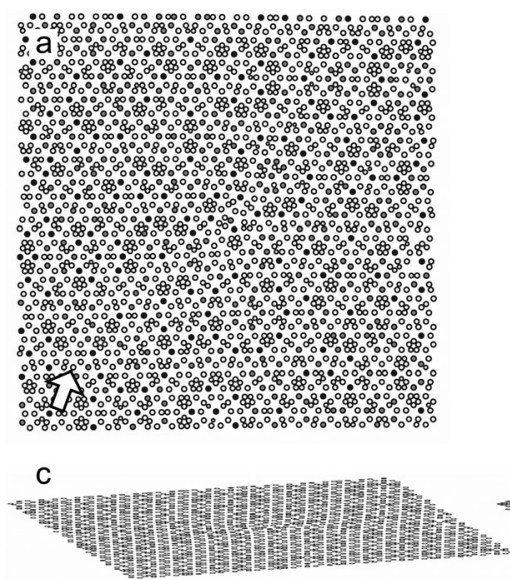

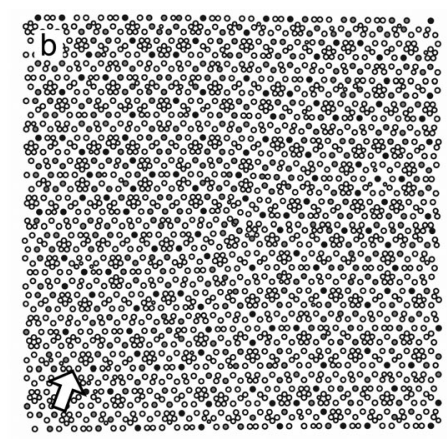

d

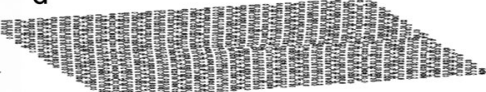

Fig. 4 Dislocation core-structure simulations in icosahedral Al-Pd-Mn. Strain field including phonon and phason components (a) and including phonon components only (b) and corresponding grazing-angle views along the arrows (c) and (d). 
Bragg-contrast imaging is usually carried out at medium resolution in the TEM, say at magnifications of 20000 to 50000 , and is a diffraction-contrast technique. ${ }^{26} \mathrm{~A}$ bright-field image is formed by electrons passing the specimen without being diffracted, contrasting with local areas in the specimen fulfilling the Bragg condition, hence diffracting electrons and appearing dark. This technique can be employed to image dislocations by choosing the imaging conditions such that only in dislocation strain fields the Bragg condition is fulfilled, so that dislocations appear as dark lines on a bright background. For an interpretable situation, the specimen orientation is chosen such that only one set of lattice planes fulfils the Bragg condition, i.e. the diffraction pattern shows only one beam besides the direct beam of undiffracted electrons. This is referred to as a two-beam condition.

Consider, in a column approximation assuming kinematic diffraction, ${ }^{27}$ a crystalline specimen illuminated by an incoming parallel electron beam with wave vector $\boldsymbol{k}_{0}$. In the column approximation we consider only the electrons diffracted by atoms in a narrow column along the incoming beam of wave vector $\boldsymbol{k}$. Atom $n$ at position $\boldsymbol{r}_{n}$ in the column contributes to the diffracted beam with a wave of amplitude $A_{p, n} \propto \exp \left(i\left(\boldsymbol{k}-\boldsymbol{k}_{0}\right)\right) \boldsymbol{r}_{n}$. The total scattering amplitude is given by the sum over all atoms in the column as

$$
A_{p} \propto\left|\sum_{\text {column }} \exp \left(i\left(\boldsymbol{k}-\boldsymbol{k}_{0}\right)\right) \boldsymbol{r}_{n}\right| .
$$

In periodic specimens, the diffraction condition $\boldsymbol{k}-\boldsymbol{k}_{0}=\boldsymbol{g}$ holds, i.e. the electrons only diffract if the scattering vector equals a reciprocal lattice vector $g$. When the column contains a defect, some atom positions are translated to positions $\boldsymbol{r}+\boldsymbol{u}$. The scattering amplitude is then subjected to an additional phase shift $\exp (-\boldsymbol{i g u})$. If a defect is present but the lattice vector and displacements are arranged such that the condition $\boldsymbol{g} \boldsymbol{u}=0$ is fulfilled no defect contrast is visible. This is referred to as the contrast-extinction condition of the defect and can, if $\boldsymbol{g}$ is known, be employed to determine the displacements $\boldsymbol{u}$.

In QCs the situation can be described analogously if we replace $\boldsymbol{g}$ by $\boldsymbol{G}=\boldsymbol{g}_{\|}+\boldsymbol{g}_{\perp}$ and $\boldsymbol{u}$ by $\boldsymbol{U}=\boldsymbol{u}_{\|}+\boldsymbol{u}_{\perp}$ (eqn (2)), where $\boldsymbol{G}$ and $\boldsymbol{U}$ are higher-dimensional vectors describing a reciprocal hyperlattice vector and the hyperlattice strain vector corresponding to the defect, respectively. The contrast of a defect in a QC is then invisible if the extinction condition $\boldsymbol{G} \boldsymbol{U}=0$ is fulfilled.

For the description of dislocations in a crystal we assume that the main component of their strain field is described by the vector $\boldsymbol{b}$. In QCs, analogously, the dislocation is described by the hyperlattice Burgers vector $\boldsymbol{B}=\boldsymbol{b}_{\|}+\boldsymbol{b}_{\perp}$ (eqn (3)). The extinction condition for dislocations in a $\mathrm{QC}$ can then be written as

$$
\boldsymbol{G B}=0,
$$

which expands to

$$
\boldsymbol{g}_{\|} \boldsymbol{b}_{\|}+\boldsymbol{g}_{\perp} \boldsymbol{b}_{\perp}=0 .
$$

This condition can be fulfilled in two different ways: ${ }^{28}$ either both parts are individually zero, or they compensate each other.
Consider the case that a dislocation is extinct when imaged using the reflection $\boldsymbol{g}_{\|}$. Then $\boldsymbol{g}_{\|} \boldsymbol{b}_{\|}+\boldsymbol{g}_{\perp} \boldsymbol{b}_{\perp}=0$ holds. Now assume that imaging with a second reflection $\boldsymbol{g}_{\|}^{\prime}=\tau \boldsymbol{g}_{\|}$in the same systematic row also leads to extinction. Then the second extinction condition $\boldsymbol{g}_{\|}^{\prime} \boldsymbol{b}_{\|}+\boldsymbol{g}_{\perp}^{\prime} \boldsymbol{b}_{\perp}=0$ also holds. Due to the one-to-one correspondence of the reciprocal lattice vectors in the perpendicular and physical subspaces, scaling the physical-space component by $\tau$ is connected ${ }^{12}$ to scaling the perpendicular-space component by $-1 / \tau$. Therefore the extinction condition for $\boldsymbol{g}_{2 \|}$ can be rewritten as $\tau \boldsymbol{g}_{\|} \boldsymbol{b}_{\|}-\tau^{-1} \boldsymbol{g}_{\perp} \boldsymbol{b}_{\perp}=0$. The two extinction conditions can only simultaneously be fulfilled if

$$
\boldsymbol{g}_{\|} \boldsymbol{b}_{\|}=0 \text { and } \boldsymbol{g}_{\perp} \boldsymbol{b}_{\perp}=0 .
$$

If we find extinction for more than one reflection in a systematic row we can hence conclude that both terms in eqn (5) individually equal zero. This is referred to as the strong extinction condition (SEC).

The second case, where the two terms in eqn (5) compensate, i.e.

$$
\boldsymbol{g}_{\|} \boldsymbol{b}_{\|}=-\boldsymbol{g}_{\perp} \boldsymbol{b}_{\perp},
$$

for a given Burgers vector can only be fulfilled by a specific reflection in a systematic row. This is referred to as the weak extinction condition (WEC). ${ }^{28}$

For the experimental Burgers vector analysis in an icosahedral QC, one has to find two SECs and one WEC. With the two SECs, the direction of the Burgers vector in physical space is determined in complete analogy with the procedure for ordinary crystals. ${ }^{27}$ The additional WEC provides information on the length ratio of the physical-space and perpendicularspace components, i.e. it determines the direction of the 6-dimensional Burgers vector in hyperspace. Obviously the existence of the WEC is a salient effect of QCs, where the phonon strain field is exactly cancelled by the phason strain field.

Since the contrast-extinction techniques described are based on orthogonality conditions, they only allow for the determination of Burgers vector directions. If the Burgers vector length is also to be determined, further solutions of the scalar product eqn (4) of the form

$$
\boldsymbol{G B}=n
$$

have to be taken into account. This can be done by analyzing multiple dislocation contrast, e.g. double contrast for $n=2,{ }^{29}$ or by means of convergent beam electron diffraction (CBED).

In CBED instead of a parallel illumination, a converging electron beam is used. For dislocation analysis, the lens is defocused, i.e. the beam crossover is placed slightly above or below the sample such that a small sample volume is illuminated. The reflections in the diffraction pattern are then enlarged to disks, the size of which is proportional to the opening angle of the illuminating beam. The sample is oriented such that the cone contains a set of beams fulfilling a specific Bragg condition. In the diffraction pattern, the set of diffracted beams forms a bright excess line, which is oriented parallel to the trace of the set of lattice planes in Bragg orientation. The excess line corresponds to a dark deficiency line in the direct disk. 
Since the crossover of the illuminating cone is placed outside the sample each beam direction belongs to a different position in the sample plane. Therefore, the diffraction disk provides a map of Bragg-plane orientations. If a defect is localized within the illuminated volume, the location of the areas where the Bragg condition is fulfilled can be slightly shifted, which is visible in the deficiency and excess lines: the lines are distorted and display splittings, which can be employed for quantitative defect characterization. Dislocations in QCs can be analysed by counting the number of splitting nodes $n$ according to the Cherns-Preston rule, ${ }^{30}$ which was generalized for QCs by Wang and $\mathrm{Dai}^{31}$ as

$$
\boldsymbol{g}_{\|} \boldsymbol{b}_{\|}+\boldsymbol{g}_{\perp} \boldsymbol{b}_{\perp}=n .
$$

Determination of all components of the Burgers vector in an icosahedral QC requires analysis of six linearly independent lines.

A typical CBED pattern is shown in Fig. 5. The direct disk (left) contains two deficiency lines (black arrows), which show threefold and twofold splitting. These splittings can also be observed in the corresponding excess lines (grey arrows).

High-resolution TEM can be employed for real-space analysis of Burgers vectors. This requires a dislocation imaged in end-on orientation and only allows for the determination of the edge components, which are perpendicular to the direction of view. The approach was further developed by Yang et al., ${ }^{32}$ who imaged dislocations by means of high-resolution TEM and characterized the image by means of a Fourier backtransform or lattice-fringe analysis ${ }^{33}$ using selected reflections $\boldsymbol{G}_{\mathrm{i}}$. For Fourier back-transforms the relation $\boldsymbol{G}_{\mathrm{i}} \boldsymbol{B}=n_{\mathrm{i}}$ holds, where $n_{\mathrm{i}}$ is the apparent number of inserted fringes using the reflection $\boldsymbol{G}_{\mathrm{i}}$. It is a particular feature of QCs that, using reflections of a single systematic row, the inserted fringes can occur on different sides of the dislocation core. This is due to the presence of the perpendicular space components of $\boldsymbol{G}$ and $\boldsymbol{B}$, and was interpreted by Bonneville et al. $^{34}$ as lattice distances of different thickness, balanced against each other for the thickness corresponding to the actual Burgers vector, which scale with different spatial frequencies. Inserted fringes on different sides of the dislocation core correspond to different signs of $n_{\mathrm{i}}$ according to a consistent sign criterion. ${ }^{32}$

For a full determination of the Burgers vector in an icosahedral QC, analysis of fringe patterns corresponding to

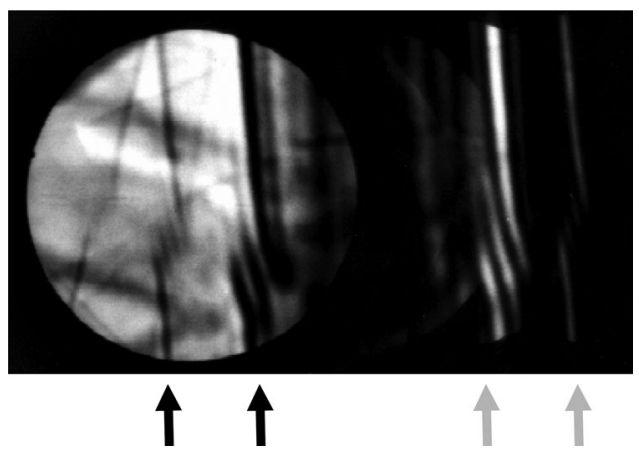

Fig. 5 Convergent-beam electron diffraction pattern displaying three- and twofold line splittings in the deficiency lines (black arrows) and excess lines (grey arrows) due to the presence of a dislocation in the illuminated specimen volume.

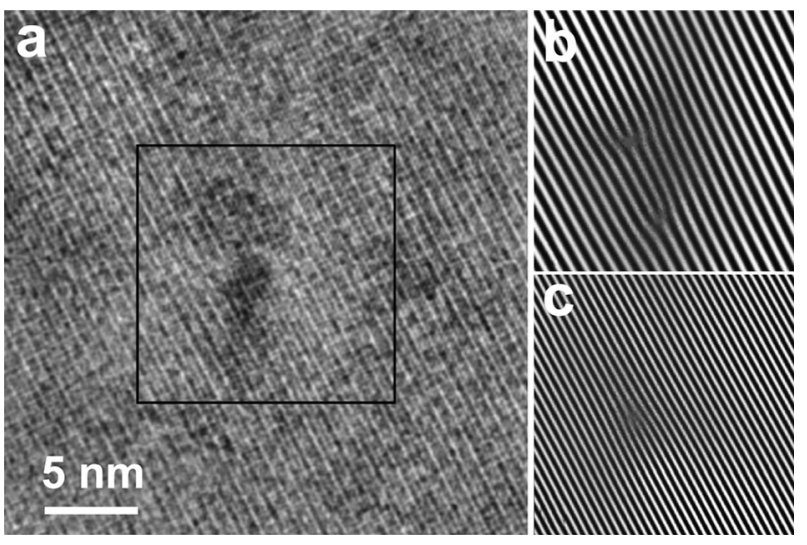

Fig. 6 Burgers vector determination by HRTEM: micrograph along a pseudo-twofold zone axis (a), back transformed using reflection $\boldsymbol{g}$ (b) and reflection $\tau \boldsymbol{g}(\mathrm{c})$. The back transformed image displays 2 and -1 inserted fringes, respectively.

six linear independent reflections is required. In a single HRTEM image, however, only four independent $\boldsymbol{G}_{\mathrm{i}}$ are available. Therefore, at least two images of the dislocation, taken at different specimen orientation are required. In both images the dislocation should be imaged as close as possible to an end-on orientation in order to obtain HRTEM images of sufficient quality to identify unambiguously the number of inserted fringes in the back transform. This is possible in icosahedral QCs due to their high structural symmetry. Dislocations with a line direction along a threefold or pseudo-twofold axis are tilted with respect to the next zone axis that can be used for HRTEM imaging by only about 11 degrees (see Fig. 1c). At a typical sample thickness of $5 \mathrm{~nm}$ this tilt leads to a smearing out of the core image over about $1 \mathrm{~nm}$, which is acceptable if an area of about $10 \mathrm{~nm} \times 10 \mathrm{~nm}$ around the core is analysed.

An example ${ }^{32}$ is shown in Fig. 6. The HRTEM image imaged along a pseudo-twofold zone axis (a) is back transformed using a reflection $\boldsymbol{g}(\mathrm{b})$ and a reflection $\tau \boldsymbol{g}(\mathrm{c})$, which are in the same systematic row but differ in length by a factor of $\tau$. The corresponding fringe patterns show 2 and -1 inserted fringes, respectively. The full analysis leads to the 6-dimensional Burgers vector $a_{0}\left[\begin{array}{llllll}\overline{3} & 2 & 2 & 0 & 0 & \overline{3}\end{array}\right]$, which has a twofold physicalspace direction and a SAC of $\tau^{7}$.

\section{Experimental dislocation analysis - icosahedral Al-Pd-Mn}

Fig. 7 displays a typical microstructure of a deformed icosahedral Al-Mn-Pd QC in a two-beam Bragg contrast image. The single quasicrystalline sample was uniaxially deformed at $760{ }^{\circ} \mathrm{C}$ up to a plastic strain of $6 \%$. The dislocation density amounts to about $10^{10} \mathrm{~cm}^{-2}$. The dislocation arrangement is homogeneous and diverse line directions occur, which corresponds to the high structural isotropy of the material.

For icosahedral Al-Pd-Mn, detailed analyses of microstructure, dislocation density and Burgers vectors were carried out. ${ }^{20,35,36}$ TEM analysis involving CBED in a great number of samples deformed at 760 and $800{ }^{\circ} \mathrm{C}$ and undeformed reference samples revealed that $90 \%$ of the dislocations have 


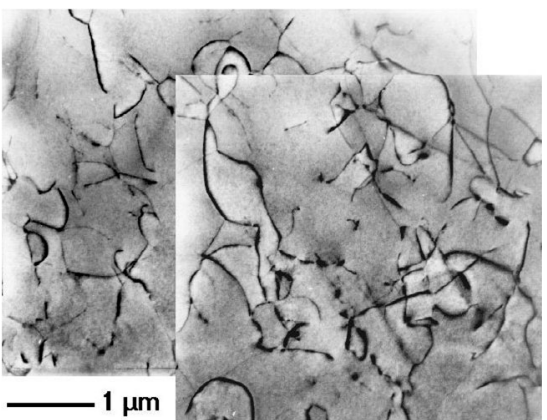

Fig. 7 TEM micrograph of a uniaxially deformed Al-Pd-Mn single QC. The image is a composite of two overlapping micrographs to enlarge the field of view.

a Burgers vector parallel to a twofold QC lattice direction. Less frequently fivefold $(5 \%)$ and threefold $(3 \%)$ and other $(2 \%)$ Burgers vectors are found. All fivefold, threefold and pseudo-twofold Burgers vectors observed are not lattice vectors in hyperspace, i.e. the corresponding dislocations are hyperspacepartials. Later studies, employing contrast extinction and multiple-contrast techniques ${ }^{25,37}$ and low-temperature deformation studies ${ }^{38}$ are consistent with the results presented. Mompiou et al $^{38}$ found pairs of dislocations, with fivefold $\left[\begin{array}{lll}1 / 0 & 0 / 1 & 0 / 0\end{array}\right]$ Burgers vectors. These correspond to [100000] hyperspace dislocations, which are not translations of the 6-dimensional face-centred lattice but only of the primitive cubic sub-lattice. In complete analogy with the situation in crystalline face-centred ordered intermetallics the authors identified these dislocations as superpartials.

For a given physical-space Burgers vector direction, several Burgers vector lengths are experimentally observed, which correspond to different 6-dimensional Burgers vectors. They are characterized by different strain accommodation parameters $\zeta$, which take different values that can be expressed as powers of $\tau$. In Table 1 the twofold Burgers vectors experimentally observed $\mathrm{d}^{20,35,39,40}$ are listed.

For all dislocations with Burgers vector parallel to a twofold quasilattice direction (referred to as twofold Burgers vectors), the exponent $n$ of the SAC is an odd integer (see Section 2 and Fig. 3). The SAC is always much larger than one, which indicates that for the dislocations it is more favourable to accommodate the displacement field through phason strain than by phonon strain.

The length scale in perpendicular space is fixed by the extension of the hypersurfaces. Therefore, the physical meaning of the phonon component of the Burgers vectors is judged by comparison of the length of $\boldsymbol{b}_{\perp}$ with the latter. In icosahedral Al-Pd-Mn, the hypersurface extensions were determined to range between $0.3 \mathrm{~nm}$ and $1.0 \mathrm{~nm} .{ }^{14}$ All values of $\boldsymbol{b}_{\perp}$ experimentally

Table 1 Twofold Burgers vectors of dislocations in icosahedral Al-Pd-Mn. $a_{0}=0.645 \mathrm{~nm}$ is the lattice parameter of the $6 \mathrm{D}$ hyperlattice, $a^{\prime}=a_{0} / \sqrt{2(2+\tau)}$

\begin{tabular}{lllll}
$\zeta$ & $\boldsymbol{B}$ & $\boldsymbol{b}_{\|}$ & $\left|\boldsymbol{b}_{\|}\right| / \mathrm{nm}$ & $\left|\boldsymbol{b}_{\perp}\right| / \mathrm{nm}$ \\
\hline$\tau^{3}$ & $a_{0}\langle\overline{1} 1100 \overline{1}\rangle$ & $a^{\prime}\langle\overline{1} / 12 / \overline{1} 1 / 0\rangle$ & 0.296 & 1.254 \\
$\tau^{5}$ & $a_{0}\langle\overline{2} 1100 \overline{2}\rangle$ & $a^{\prime}\langle\overline{2} / 13 / \overline{2} 1 / \overline{1}\rangle$ & 0.183 & 2.030 \\
$\tau^{7}$ & $a_{0}\langle\overline{3} 2200 \overline{3}\rangle$ & $a^{\prime}\langle\overline{3} / 25 / \overline{3} 2 / \overline{1}\rangle$ & 0.113 & 3.281 \\
$\tau^{9}$ & $a_{0}\langle\overline{5} 3300 \overline{\overline{5}}\rangle$ & $a^{\prime}\langle\overline{\overline{5}} / 38 / \overline{5} 3 / \overline{2}\rangle$ & 0.070 & 5.321 \\
\hline
\end{tabular}

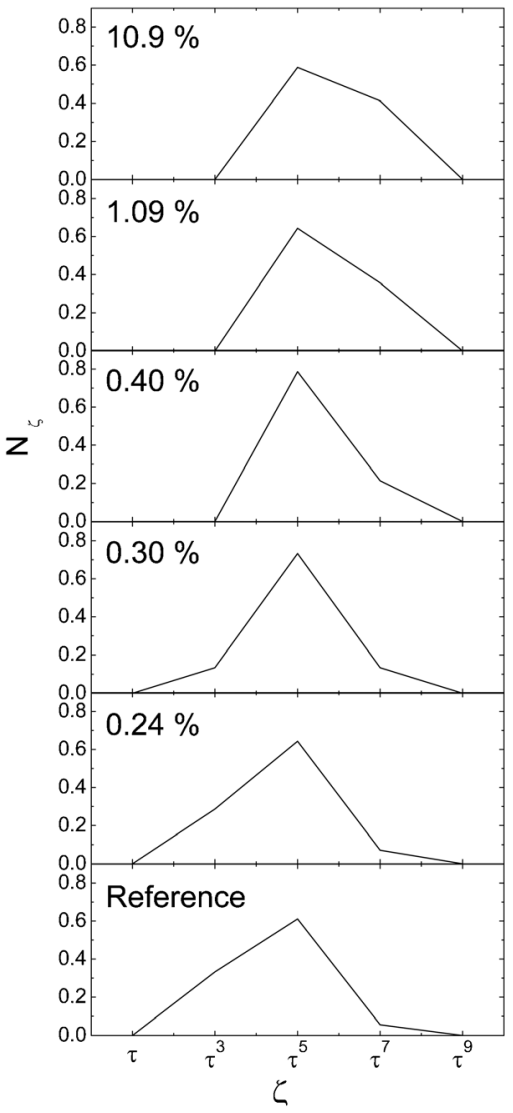

Fig. 8 Normalized frequency of occurrence $N_{\zeta}$ of twofold Burgers vectors with a certain strain accommodation parameter for undeformed material (reference) and samples plastically deformed at $760{ }^{\circ} \mathrm{C}$ between $0.24 \%$ and $10.9 \%$.

observed surpass this size, most by a considerable factor. This indicates that the dislocations should be accompanied by a large number of phasons, which is in accordance with the experimental results (see below).

Dislocations with different SAC are found at different densities in the material. Dislocations with an SAC of $\tau^{5}$ most frequently occur and the other types follow a distribution curve which drops rapidly for higher and lower values. The distribution curve depends on the degree of plastic deformation of the sample. Fig. 8 shows the normalized frequency of occurrence $N_{\zeta}$ of twofold Burgers vectors with a certain strain accommodation parameter for undeformed material (reference) and samples plastically deformed at $760{ }^{\circ} \mathrm{C}$ between $0.24 \%$ and $10.9 \%$. The maximum of all distribution curves remains at $\tau^{5}$, i.e. for all deformations Burgers vectors with a parallelspace length of 0.183 occur most frequently. However, the shape of the curve changes, and the center of weight clearly shifts to higher average SAC with increasing plastic deformation. The average character of the dislocations thus changes with increasing strain towards a larger phason component. A similar result for samples deformed at $800{ }^{\circ} \mathrm{C}$ study was obtained by Rosenfeld et al. ${ }^{35}$ These authors also demonstrated that the Burgers vector distribution does not depend on the deformation temperature.

It has been demonstrated by Wang et al. ${ }^{41}$ and Wollgarten et al. ${ }^{42}$ that the change of average dislocation character towards 
higher phason components can arise due to dislocation reactions taking place during the deformation process. The authors demonstrate that reactions transferring dislocations with low SAC to dislocations with higher SAC at constant physical-space orientation are in all cases energetically favourable. For example, a reaction transferring a twofold dislocation of type $\tau^{3}$ into two twofold dislocations of type $\tau^{5}$ and $\tau^{7}$ is found, which can account for the distribution change in Fig. 8. It is notable that in this reaction, for the initial dislocation a fraction of about $5 \%$ of the total energy is stored in the phason component, while for the product dislocations a value of about $30-40 \%$ is found.

The Burgers vector results compare well to findings in the icosahedral phase of the system $\mathrm{Al}-\mathrm{Cu}-\mathrm{Fe}$. Dislocations of type $\tau^{3}$ with twofold $a_{0}\langle\overline{1} 1100 \overline{1}\rangle$ Burgers vectors ${ }^{43-45}$ as well as threefold and fivefold Burgers vectors were identified. ${ }^{46}$ Since in these studies only individual dislocations were characterized, no conclusions on the relative frequencies of occurrence of different types of Burgers vector can be drawn.

In the early microstructural studies, ${ }^{20,35,47}$ the mode of dislocation motion was interpreted in terms of glide. This is not due to factual opinion but to historical reasons. In crystalline materials for the vast majority of cases dislocations are known to move by pure glide, while only in a few exceptions climb is found. Therefore it was initially obvious and natural to discuss QC plasticity in terms of glide. Accordingly, apparent glide planes were determined as the plane spanned by the dislocation line direction and the Burgers vector. First indications calling these analyses into question were found by Wang et al., ${ }^{48}$ who analyzed planar faults, trailed by dislocations, and found that the fault normals were parallel to their displacement vectors. A dedicated study by Caillard et al. ${ }^{49}$ involving a fringe-contrast analysis later unambiguously established that dislocations in icosahedral Al-Pd-Mn at high temperatures move by pure climb, i.e. the Burgers vector is perpendicular to the plane of dislocation motion. This was later confirmed in several studies ${ }^{34,37}$ and also interpreted in terms of tiling models. It was shown ${ }^{25}$ that glide motion of dislocations in a tiling creates heavily distorted zones that cannot be retiled with the tiles available. On the other hand climb motion only leads to the removal or insertion of "worms" (the tiling equivalent to a crystal lattice plane), which does not involve new tiles and can be interpreted in terms of phason defects. In low-temperature deformation experiments under high-pressure confinement ${ }^{38}$ pure climb dislocation movement was demonstrated at $300{ }^{\circ} \mathrm{C}$.

Nevertheless, there are still open questions concerning the mode of dislocation motion in icosahedral QCs. First, in icosahedral Al-Pd-Mn high-impact deformation experiments ${ }^{48}$ revealed Burgers vectors in the plane of dislocation motion indicating glide motion under these particular conditions of high strain rate and low-deformation temperature, and glide was also found in low-temperature deformation experiments in a shear cell. ${ }^{50}$ Second, in icosahedral $\mathrm{Zn}-\mathrm{Mg}$-Dy quasicrystals, glide is identified as the prominent deformation mode. This will be discussed in more detail in Section 7.

In summary, under regular controlled deformation conditions in icosahedral Al-Pd-Mn, dislocations most frequently have twofold Burgers vectors with a physical-space length of $0.183 \mathrm{~nm}$.
The dislocations move by climb on twofold, threefold and fivefold planes. $^{34}$ In in-situ experiments ${ }^{37,51}$ straight dislocation segments along twofold and pseudo-twofold directions are found. This indicates structural anisotropy, which was interpreted in terms of Peierls-valley models ${ }^{52,53}$ and cluster friction. ${ }^{20}$ The cluster-friction model accounts for the characteristic features of high-temperature QC plasticity, and can in a constitutive-equation formulation ${ }^{54}$ also quantitatively account for the experimentally observed work softening and dislocation density evolution. ${ }^{55}$

\section{Phasons}

In the previous section we have seen that the average phason component of dislocations in deformed icosahedral Al-Pd-Mn increases with increasing plastic strain. This indicates that phason defects are involved in the deformation process. It is therefore useful to investigate the role of phasons in QC plasticity.

\section{Phasons in reciprocal space}

In a linear approximation phason-type displacement fields $\Delta \boldsymbol{x}_{\perp}$ can be described by ${ }^{56}$

$$
\Delta \boldsymbol{x}_{\perp}=-\boldsymbol{M}_{\perp}^{T} \cdot \boldsymbol{x}_{\|}
$$

where $\boldsymbol{x}_{\perp}$ is the physical space coordinate, $\boldsymbol{M}_{\perp}$ is a $3 \times 3$ matrix, the so-called phason matrix, and the superscript $T$ denotes transposition of the matrix. The displacement fields described by (7) lead to deviations $\Delta \boldsymbol{g}_{\|}$of the positions of diffraction spots in reciprocal space according to ${ }^{57}$

$$
\Delta g_{\|}=M_{\perp} \cdot g_{\perp},
$$

where $g_{\perp}$ is the perpendicular-space component of the reciprocal lattice vector. In order to understand the consequences of (8) for electron diffraction patterns of phason strained QCs, let us consider the construction of the QC reciprocal lattice by means of higher-dimensional crystallography.

In a kinematic approximation the diffraction pattern can be calculated as the Fourier transform of the direct hypercrystal, i.e. the direct hyperlattice and its decoration. The transformation of the direct hyperlattice leads to a reciprocal hyperlattice, which is convoluted with the transformation of the atomic hypersurfaces. In the simplest form of the intersection procedure, we use a box function for the description of the hypersurfaces. Its Fourier transform is then a sinc function, i.e. of the form $\sin (x) / x$. Therefore, the reciprocal hypercrystal consists of a higher-dimensional lattice, where each lattice point is decorated by a sinc function. This is schematically represented for the 1-dimensional case in Fig. 2d. The QC diffraction pattern is constructed in analogy to the direct crystal: a second rotated coordinate system, defining reciprocal physical space and reciprocal perpendicular space is introduced, and the intersections of the Fourier transformed hypersurfaces, which have an extension only in reciprocal perpendicular space, with reciprocal physical space defines the QC reciprocal lattice points. Note that, since the sinc function extends infinitely, this procedure yields an infinitely dense occupation of reciprocal lattice points. This is obviously 
in agreement with the fact that a QC in direct space has an infinitely large periodicity.

Experimentally, nevertheless a discrete diffraction pattern is obtained, since the intensity of the reflections is weighted by the function value of the Fourier transformed hypersurfaces at the intersection. The envelope of the sinc function, here $\sin \left(q_{\perp}\right) / q_{\perp}$, decreases as $1 /\left|q_{\perp}\right|^{2}$, where $q_{\|}$and $q_{\perp}$ are the respective coordinates in reciprocal space. Therefore, the reciprocal lattice points, corresponding to a small $q_{\perp}$ exhibit a reflection with a high intensity, while those corresponding to very a high $q_{\perp}$, which is obviously the case for the majority of reciprocal lattice points, exhibit reflection intensities so low that they cannot be recorded within normal laboratory timescales. Fig. 2d shows the construction of the diffraction pattern corresponding to the 1-dimensional QC of Fig. 2a. The radius of the circles representing the reflections corresponds to the respective intensity.

With these considerations and eqn (8) we understand that the presence of phason strain causes characteristic reflection shifts in electron diffraction patterns. The reflections with the smallest intensities correspond to the highest $\boldsymbol{g}_{\perp}$ and thus suffer the strongest shifts. The shifts can be detected as deviations from the perfect alignment along straight systematic rows, for example by inspecting electron diffraction patterns at grazing view. A zig-zagged arrangement of spots occurs, which can be regarded as fingerprint behaviour of phason strain.

Fig. 9 depicts sections of a diffraction pattern of icosahedral $\mathrm{Al}-\mathrm{Pd}-\mathrm{Mn}$ in grazing view. Fig. 9a and b show systematic rows of an undeformed reference sample and a sample

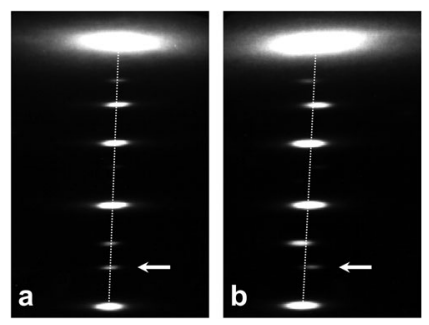

Fig. 9 Systematic row in a diffraction pattern of undeformed (a) and plastically deformed (b) icosahedral Al-Pd-Mn. In (b) deviations from the ideal spot positions due to the presence of phason strain are seen. The dotted line is a guide to the eye, indicating the course of the ideal systematic row. deformed by $0.25 \%$ at $760{ }^{\circ} \mathrm{C}$, respectively. In the undeformed reference sample, all reflections are perfectly aligned along the systematic row (dotted line). In the deformed sample, on the other hand, clear deviations from the systematic rows are seen, forming a zig-zagged arrangement of reflections. Obviously the spots with the lowest intensities suffer the strongest shifts, which, as described above, is a distinguishing feature revealing the presence of phason strain in the material. The arrows indicate the ideal position in (a) and the deviated position in (b) of a weaker spot.

The deviation $\Delta \boldsymbol{g}_{\|}$of the reflections can be measured quantitatively by comparison with a calculated ideal diffraction pattern. The results for a single systematic row are depicted in Fig. 10. In the leftmost panel the magnitude of the measured spots shifts $\left|\Delta \boldsymbol{g}_{\|}\right|$in an undeformed reference sample are plotted against the magnitude of their perpendicular-space reciprocal vector $\left|\boldsymbol{g}_{\perp}\right|$. The shifts are very small, which corresponds with the visual inspection (Fig. 9a). The middle panel shows the same for reflections in a sample deformed by $0.25 \%$. The shifts are significantly larger than in the reference sample, and their amount linearly depends on $\left|\boldsymbol{g}_{\perp}\right|$. If the same shifts are plotted against the magnitude of their physical-space reciprocal vector $\left|\boldsymbol{g}_{\|}\right|$(rightmost panel), no linear dependence but a rather unsystematic behaviour is found. These results show that indeed linear phason strain is present in the deformed sample, and the calculation of the phason matrix is possible according to eqn (8). A typical result for a phason matrix is

$$
\boldsymbol{M}_{\perp}=10^{-3} \cdot\left(\begin{array}{ccc}
6.9 & -1.0 & -0.8 \\
-0.5 & 1.2 & -1.1 \\
M_{31} & M_{32} & M_{33}
\end{array}\right)
$$

Note that it is generally impossible to determine the full matrix by this method, since the diffraction patterns only allow for the detection of the shifts perpendicular to the direction of view. According to the choice of the coordinate system, which is defined by the parallel orientation of the foil normal and the $(0 / 00 / 00 / 2)$ direction, it is impossible to determine the third row of the matrices $\boldsymbol{M}_{\perp}$. Measurements in different sample areas and along different systematic rows yield matrices which have comparable entries within the measurement accuracy of the experiment. Significant is the entry $M_{11}$, which varies between 5.7 and 9.1 in different experiments and takes an average value of 7.23 .

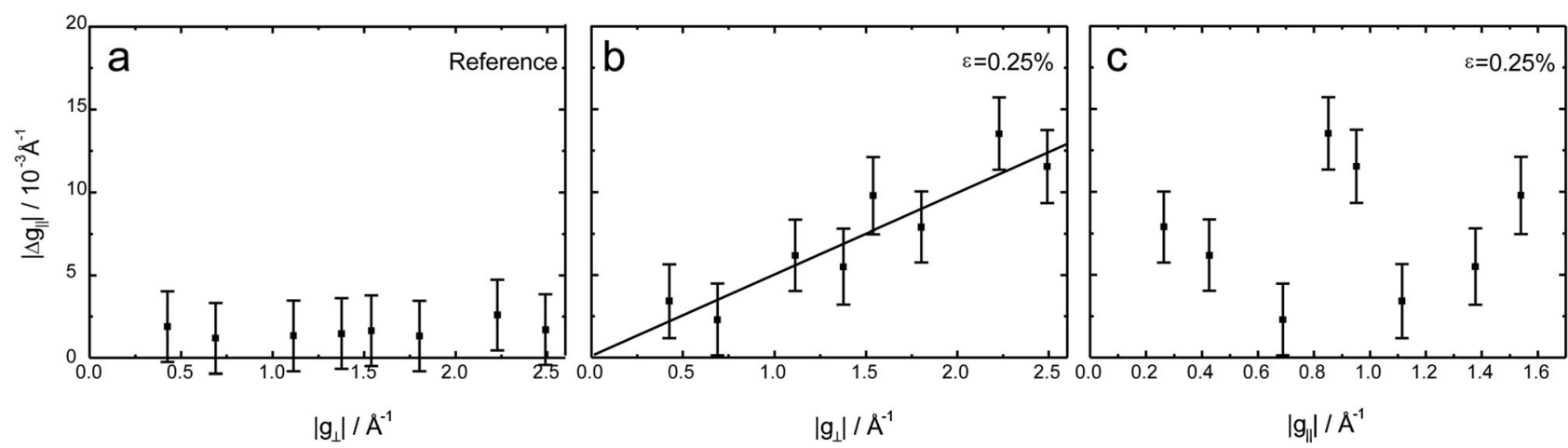

Fig. 10 Spot shifts $\left|\Delta \boldsymbol{g}_{\|}\right|$in undeformed (a) and deformed (b) and (c) icosahedral Al-Pd-Mn plotted as a function of perpendicular-space reciprocal vector $\left|\boldsymbol{g}_{\perp}\right|$ (a) and (b) and physical-space reciprocal vector $\left|\boldsymbol{g}_{\|}\right|$(c). The solid line in (b) represents the linear increase of $\left|\boldsymbol{g}_{\perp}\right|$ corresponding to the phason matrix $\boldsymbol{M}_{\perp}$. 
The observed spot shifts and the phason matrices determined show a clear dependence on the amount of plastic deformation. The undeformed reference samples, as shown above, do not show visible spot shifts. Accordingly the entries in the phason matrix are small and the entry $M_{11}$ is zero. A sample deformed by $0.15 \%$ shows visible spot shifts. The phason matrix has entries in between those of the reference sample and that deformed by $0.25 \%$ and we find $M_{11}=2.3$.

It is apparent that the phason matrix determined is not symmetric. Matrices describing the transformation of icosahedral symmetric structures into tetrahedral, pentagonal or trigonal symmetry have been explicitly given by Ishii. ${ }^{58}$ Owing to the experimental uncertainties, it is not possible to decide whether the matrices determined can be identified with those described by Ishii.

\section{Phasons in direct space}

Phason defects can be observed in direct space by means of highresolution TEM. Fig. 11a is a high-resolution scanning TEM micrograph of icosahedral $\mathrm{Al}-\mathrm{Pd}-\mathrm{Mn}$ along a fivefold direction.

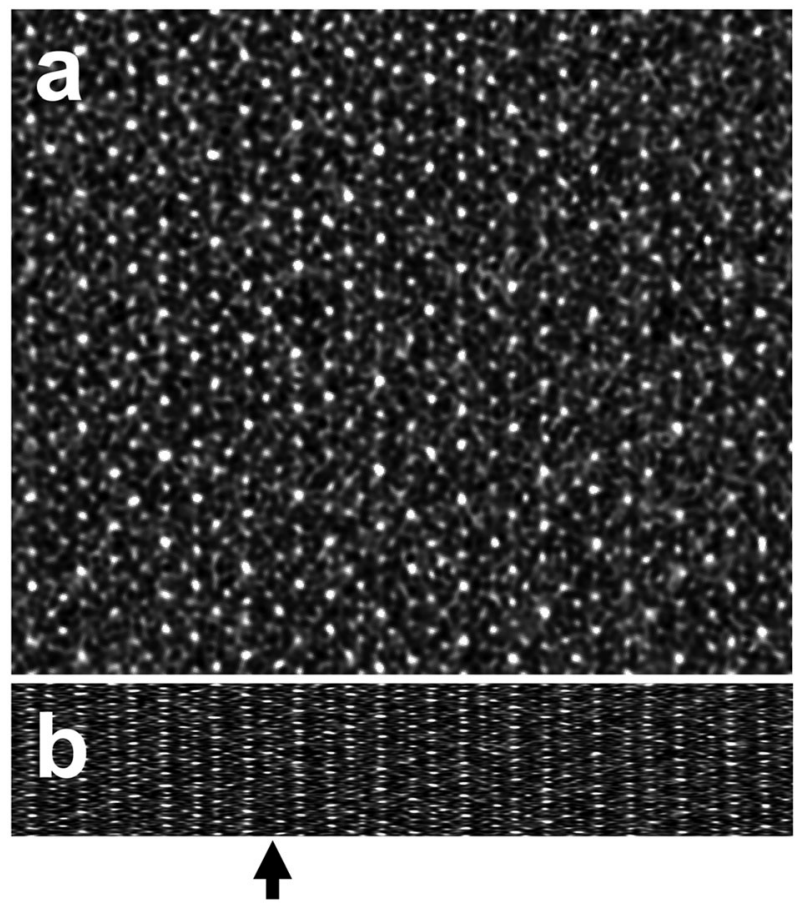

Fig. 11 High resolution scanning TEM micrograph along a fivefold direction displaying jags (arrow) due to the presence of phason strain (a); the same area in grazing view along the vertical image direction (b).
Close inspection of the image reveals that some lattice planes do not continue throughout the image but fade out and are continued by neighboured lattice planes that fade in. This can be better seen at a grazing angle as in Fig. 11b. One example of a discontinuous lattice plane is marked by an arrow. These defects are referred to as jags and can straightforwardly be interpreted in terms of phasons: in an ideal QC each set of lattice planes follows a sequence of short and long distances, the order of which follows a Fibonacci sequence. A jag in a lattice plane locally changes the order, and leads to a deviation from the ideal sequence, which directly complies with the definition of a phason. In high-resolution TEM images of dislocations, jags surrounding the core corresponding to the phason component of the Burgers vector are found (Section 3, Fig. 4c).

Planar accumulations of phasons can also lead to the occurrence of characteristic fringes in diffraction-contrast TEM. Such a situation can be found in the wake of dislocations that have moved at relatively low temperatures. Fig. 12a shows a bright-field Bragg-contrast image of a planar defect imaged using a $(\overline{1} / 00 / 10 / 0)$ reflection (inset). During the growth process of the $\mathrm{QC}$, the material is subjected to thermal stresses. This induces some amount of dislocation motion, which under these conditions leads to the creation of planar defects. Their contrast closely resembles that of an ordinary stacking-fault, but by suitable in situ annealing experiments it can be shown that the faults consist of a planar arrangement of a high local density of phasons.

Fig. 12b shows the same specimen area as in (a) after heating the specimen to $580{ }^{\circ} \mathrm{C}$. The surface of the specimen now shows a cloudy contrast due to degradation of the sample during heating. Nevertheless, the fringe contrast of the fault as well as the leading dislocation (arrow) are still clearly visible. When the heat treatment is resumed over longer durations, the fringe contrast gradually weakens and eventually disappears. This process does not involve any dislocation motion or size changes of the contrast. Fig. 12c shows the same specimen area imaged under the same conditions and with the same diffraction vector (inset) after $100 \mathrm{~min}$ at $580{ }^{\circ} \mathrm{C}$. The fringe contrast has entirely vanished. Quantitative measurements show that the contrast decays exponentially with a temperature dependent time constant, which in the present case is about 1000 seconds.

During vanishing of the fringes, the contrast and position of the dislocations remain completely unchanged. An ordinary stacking fault or similar structural defect cannot disappear without movement of the trailing dislocations and the disappearance
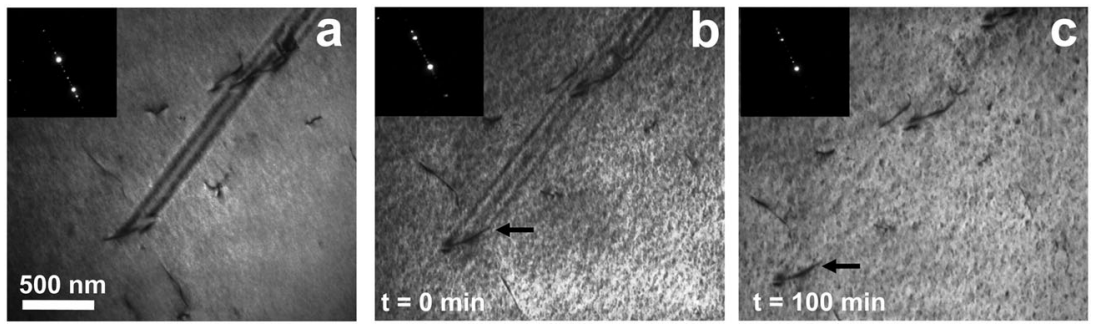

Fig. 12 Bright-field Bragg-contrast image of a planar defect in icosahedral Al-Pd-Mn (a), the same specimen area after heating the specimen to $580{ }^{\circ} \mathrm{C}$ (b) and after 100 minutes at $580{ }^{\circ} \mathrm{C}$ (c). 
cannot appear gradually. The observed particular contrast behavior upon annealing can only occur since the planar faults consist of an agglomeration of phason defects, and can be dissolved by essentially independent movement of the latter into the bulk. ${ }^{59}$

The introduction of phasons by moving dislocations is straightforwardly related to the quasiperiodicity of the material. In any ordered nonperiodic structure, the motion of a dislocation for strictly geometrical reasons must alter the structure in its wake: due to the lack of translational symmetry, the Burgers vector of a dislocation cannot be translationally invariant and hence, upon its movement, a planar defect must be created. The nature of the defect created for the case of quasicrystals was described by Socolar et al., ${ }^{9}$ who demonstrated that an agglomeration of local matching-rule violations is introduced in the plane swept over by a dislocation. In other words, a moving dislocation in a quasicrystal creates a plane of phason defects. This finding was confirmed by molecular dynamics simulations. ${ }^{60}$ Independent experimental characterizations of the planar faults have revealed their phason-type nature. The matching-rule violations along lattice planes perpendicular to the plane of dislocation motion were directly imaged by high-resolution $\mathrm{TEM}^{48}$ and dedicated fringe-contrast analysis experiments ${ }^{49}$ unambiguously demonstrated the phasonic nature of the faults.

The exponential disappearance of the contrast shows that the underlying mechanism is a first order reaction, which indicates a diffusive dissolution of the faults. We can therefore draw the following conclusions: at very low temperatures the kinetics of the diffusion process is low, so that the phason defects introduced remain essentially within the plane of dislocation motion. The situation then corresponds to the dislocation simulation depicted in Fig. $4 \mathrm{~b}$ and $\mathrm{d}$ and the phason defects can directly be imaged by TEM as fringe contrasts (Fig. 12a and b). At high temperatures, on the other hand, the phason-diffusion kinetics becomes so fast that no permanent defect planes are created. The defects immediately diffuse into the bulk, and the moving dislocation is locally surrounded by an increased phason density. The situation then corresponds to the dislocation simulations depicted in Fig. 4a and $\mathrm{c}$ and the phason defects cannot be directly imaged by TEM. Dislocations appear as single linear defects without attached planar defect (Fig. 7). Nevertheless, phason defects are continuously produced during dislocation motion and hence plastic deformation leads to their presence and accumulation in the structure. In specimens deformed under these conditions, phasons can be detected by reflection shifts in electron diffraction patterns (Fig. 9b).

The continuous accumulation of phason defects during dislocation motion has considerable effects on the macroscopic plastic behavior of icosahedral quasicrystals. It leads to a decreasing resistance against dislocation mobility, which, in combination with fast dislocation annihilation ${ }^{55}$ is a major ingredient of the characteristic strain-softening phenomenon of icosahedral quasicrystals.

\section{Dislocations in icosahedral $\mathbf{Z n - M g - R E}$ quasicrystals}

A new icosahedral phase was discovered by Luo et al. ${ }^{61}$ in the $\mathrm{Zn}-\mathrm{Mg}-\mathrm{Y}$ alloy system. Isostructural phases exist in

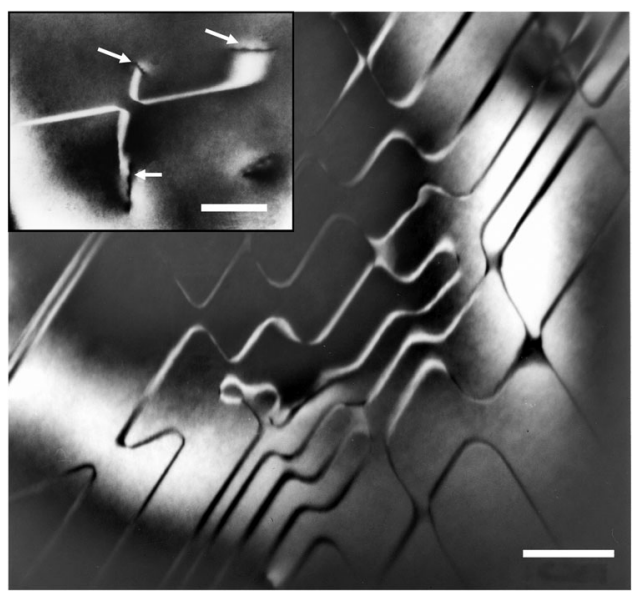

Fig. 13 Bright-field Bragg-contrast image of the typical defect structure in icosahedral deformed $\mathrm{Zn}-\mathrm{Mg}$-Dy sample. The inset shows a similar defect arrangement in a heat-treated sample, the arrows point at dislocation lines. The scale bar corresponds to $200 \mathrm{~nm}$ in the main image and $400 \mathrm{~nm}$ in the inset.

the systems $\mathrm{Zn}-\mathrm{Mg}-\mathrm{RE}$, where RE stands for the rare-earth metals Dy, Ho, Er, Tb, and Gd. As icosahedral Al-Pd-Mn, discussed above, these phases have a face-centred hyperlattice and the physical-space atomic positions can be constructed via the intersection procedure from a 6-dimensional hyperlattice. Nevertheless, substantial differences are found in the plastic behaviour and in the defect structures.

Detailed microstructural investigations were carried out on the icosahedral phase $\mathrm{Zn}-\mathrm{Mg}$-Dy. Fig. 13 shows a bright-field Bragg-contrast image of the typical defect structure in a sample deformed up to a plastic strain of about $0.2 \%$, which corresponds to the upper yield point. A high density of planar faults is observed. The planar faults could be identified as antiphase boundaries (APBs). ${ }^{62}$ The APBs are created by moving dislocations due to the particular hyperlattice structure of icosahedral $\mathrm{Zn}-\mathrm{Mg}-\mathrm{Dy}$. The inset of Fig. 13 shows a defect arrangement with clearly visible dislocation lines (arrows) terminating the APBs in a heat-treated sample, which contains similar defects at lower density.

A structure model for icosahedral $\mathrm{Zn}-\mathrm{Mg}-\mathrm{Ho}$ represented by a higher-dimensional unit cell was proposed by Ohno and Ishimasa. ${ }^{63}$ The face-centred unit cell contains five spherical hypersurfaces. The hyperlattice nodes $\mathrm{n}_{0}$ and $\mathrm{n}_{1}$ (cf. Fig. 2b) are occupied by hypersurfaces representing $\mathrm{Zn}$ and $\mathrm{Mg}$ atom positions, respectively. In contrast to the unit cell of icosahedral $\mathrm{Al}-\mathrm{Pd}-\mathrm{Mn}$, the $\mathrm{bc}_{2}$ position is also occupied and an additional edge-center position is introduced. The model is more elaborate than that of icosahedral Al-Pd-Mn but does not describe the structure as accurate. Short atomic distances in physical space occur and the overall reliability factor of the model is about $24 \%$. An improved model including an elliptical edge-center position was presented later on, ${ }^{64}$ which also did not lead to satisfactory results.

Even though the available models have their shortcomings, we can see that the hyperlattice is an ordered face centred structure. The physical-space structure therefore ${ }^{65}$ possesses corresponding site-occupation order and the diffraction patterns contain superstructure reflections along particular directions, 
in this case threefold and fivefold. The presence of the ordered structure allows for the formation of APBs as boundaries between different order domains.

The planar faults in plastically deformed icosahedral $\mathrm{Zn}-\mathrm{Mg}$-Dy (Fig. 13) can be imaged by diffraction contrast only using superstructure reflections. Using fundamental reflections they are out of contrast. This identifies them undoubtedly as APBs, and also implies that the dislocations terminating the faults correspond to hyperlattice superpartial dislocations.

The Burgers vectors of individual dislocations were determined by contrast-extinction techniques. ${ }^{66}$ In all cases, the Burgers vectors were found to be parallel to twofold directions of the QC lattice. Between the Burgers vectors and the line direction most frequently an angle of $60^{\circ}$, corresponding to the angle between twofold directions, is observed and thus the dislocations are of mixed edge/screw type. CBED or multiplecontrast experiments have not been carried out, so the length of the Burgers vectors is unknown.

In order to determine the mode of dislocation motion, the plane normals of the APBs were determined and brought into relation with the Burgers vector direction. In $60 \%$ of the cases, relative angles of $90^{\circ}$ were found in deformed samples, which shows that these dislocations move by pure glide. In another $20 \%$, angles between $75^{\circ}$ and $80^{\circ}$ were observed, which also correspond to a very high glide component. These findings are corroborated by the observation that these APB planes and the Burgers vector directions are all oriented close to a $45^{\circ}$ cone around the compression axis. The corresponding glide systems have Schmid factors close to 0.5 , i.e. the activated slip systems resolve maximum shear stress. On the other hand, in the remaining $20 \%$ of cases, angles between APB normals and Burgers vectors between $32^{\circ}$ and $64^{\circ}$ were found. These dislocations have a considerable climb component and in accordance with this observation, we found a number of APB normals parallel to the compression direction. On these planes the resolved shear stress is zero, and only dislocations moving with a climb component can contribute to strain.

Fig. 14a is a high-resolution scanning TEM micrograph of a dislocation in icosahedral $\mathrm{Zn}-\mathrm{Mg}-\mathrm{Dy}$ along the fivefold

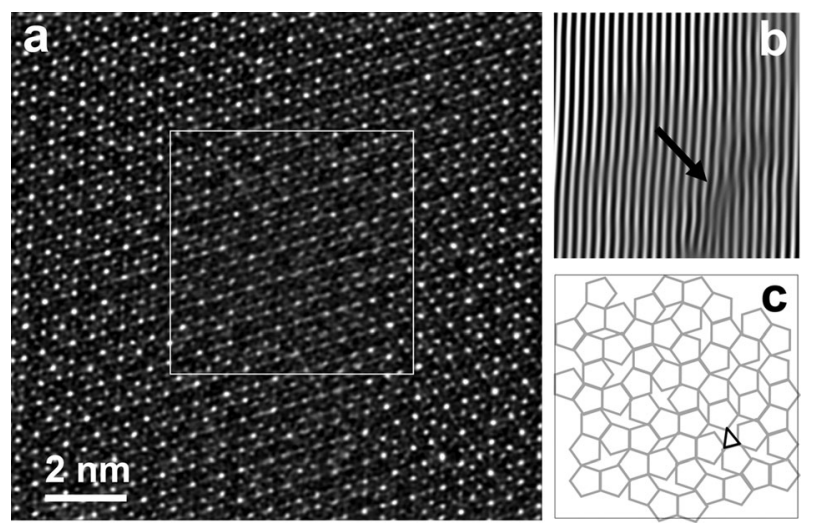

Fig. 14 High resolution scanning TEM micrograph of a dislocation in icosahedral $\mathrm{Zn}-\mathrm{Mg}$-Dy along a fivefold direction (a). Back Fourier transformed fringe pattern of the boxed area displaying an inserted fringe (b). Tiling analysis employing a rhomb-pentagon tiling (c), the dislocation core is represented by a triangle (black).
[0/0 $1 / 0$ o/1] direction. The dislocation is imaged almost end on, i.e. its line direction is approximately parallel to the electron beam. The dislocation core is located in the boxed area, where a region of deviated structure can be seen. Outside of the box, the perfect icosahedral structure is resolved. The exact position of the dislocation cannot be located due to the rather large distorted region and a slight misorientation of the line direction. Fig. $14 \mathrm{~b}$ is a fringe pattern of the boxed region in (a), obtained by back-Fourier transformation using a twofold (4/4 0/0 0/0) reflection. Clearly an inserted halfplane in the lower right corner is seen (arrow). Fig. 14c shows an analysis of the boxed region in terms of a rhomb-pentagon tiling. The tiling is started in the perfect region outside the box and continued into the distorted region. The boxed area is tiled using regular pentagons and rhombs, plus two additional tiles. The latter are boat- and crown-shaped and are topologically equivalent to pentagons, in the sense that they are constructed using the same edges as the pentagon only in a different order.§ The entire area can be covered except a small triangular area (dark lines), which corresponds to the dislocation core. When the three closest edges of an undistorted ideal pentagon are used to construct the triangle a closure failure occurs, which corresponds to the projection of the Burgers vector on the image plane. We obtain a Burgers vector projection parallel to the twofold $[\overline{1} / 01 / 10 / \overline{1}]$ direction with a physical-space length of about $0.191 \mathrm{~nm}$. This in-plane Burgers vector component is of reasonable length and corresponds well to the values found for icosahedral Al-Pd-Mn.

Finally, let us discuss the question, why APBs are observed so dominantly in icosahedral $\mathrm{Zn}-\mathrm{Mg}-\mathrm{Dy}$, while this is not the case with icosahedral Al-Pd-Mn. Both phases do have face centred hyperlattices, but the decoration of the 6-dimensional unit cell by atomic hypersurfaces is very different. In particular, for $\mathrm{Zn}-\mathrm{Mg}-\mathrm{Dy}$ the hypersurfaces on the nodes $\mathrm{n}_{0}$ and $\mathrm{n}_{1}$ correspond to different atomic species, $\mathrm{n}_{0}$ entirely to $\mathrm{Zn}$ and $\mathrm{n}_{1}$ entirely to $\mathrm{Mg}$ and the difference in their extension is about $31 \%$. In Al-Pd-Mn these nodes are rather similar: both $\mathrm{n}_{0}$ and $\mathrm{n}_{1}$ have an inner shell corresponding to $\mathrm{Mn}$, and an outer shell corresponding to $\mathrm{Al}$ and their outer radius differs only by $5 \%$. The main difference is that the hypersurface on the $\mathrm{n}_{1}$ node has an additional intermediate shell corresponding to Pd. Hence, only a small fraction of atoms in physical space is affected by these differences, and accordingly the contrast of the APBs will be weaker than in $\mathrm{Zn}-\mathrm{Mg}-\mathrm{Dy}$. However, if a superstructure reflection is used for imaging, they should be visible. Indeed, Mompiou et al. ${ }^{38}$ observed APBs between pairs of dislocations with fivefold Burgers vectors, which were identified as split superdislocations.

\section{Conclusions}

The field of dislocations in QCs has, over the past three decades, developed into a well explored and solidly founded domain of materials science. In particular the icosahedral Al-Pd-Mn phase has developed into a prominent exemplary system, rendered possible by the availability of large single

$\S$ The boat-shaped tile has seven edges, but two of these cancel as they are followed in opposite direction in a closed circuit around the edges. 
crystals of high structural quality. The microstructural as well as the macroscopic plastic properties of this phase have been well characterized, and novel mechanisms, directly related to the quasiperiodicity of the structure, have been identified. Most obvious is the contribution of phasons. Phasons are pivotally involved in the formation of dislocations, and the counteraction of their dynamics with that of the other processes taking place during deformation dominates the deformation mechanism. This leads to salient features in the macroscopic mechanical properties such as the deformationsoftening phenomenon, which discriminates the plasticity of icosahedral QCs from most other ordinary metals. In order to account for these new aspects novel concepts were developed, such as the higher-dimensional description of structure and defects, and the phason component of the Burgers vector. These concepts are generally required for the description of QC plasticity and come into play also for other types of QC, e.g. decagonal phases.

Besides icosahedral Al-Pd-Mn, only one further icosahedral QC has been extensively explored - the icosahedral phase in the system $\mathrm{Zn}-\mathrm{Mn}-\mathrm{Dy}$. In this phase, as a result of the different structure type, different phenomena are observed, e.g. the dominant presence of APBs and dislocations moving by glide. This shows that a solid understanding of the plasticity of one single QC phase is not sufficient for a general understanding of the characteristic phenomenological differences between the plasticity of QCs and periodic crystals. Hence, the approach of further QC phases is essential for future research. In particular this concerns the new class of binary and pseudo-binary icosahedral phases based on $\mathrm{CdY},{ }^{67}$ which is based on a different cluster type. Also fully unaddressed concerning plastic properties and defects are one-dimensional QCs, which structurally consist of a quasiperiodic stacking of periodic planes. Stable phases of this class of QCs have e.g. been found in the $\mathrm{Al}-\mathrm{Pd}-\mathrm{Fe}$ alloy system. ${ }^{68}$

In recent years, the concepts developed for the description of QC defects and plasticity have turned out to be extremely useful for the approach of complex metallic alloys (CMAs). These materials are periodic, and their structure is characterized by a high number of atoms per unit cell. ${ }^{69}$ They are closely related to QCs in the sense that their local order is organized in the form of icosahedral clusters, which in some cases are almost identical to those in corresponding QCs. In the CMA phase $\varepsilon-\mathrm{Al}-\mathrm{Pd}-\mathrm{Mn}$ a novel type of defect, referred to as metadislocation, was discovered, ${ }^{70}$ which could only be adequately described by applying the concepts of QC plasticity: metadislocations are associated to phason defects, which are involved in their formation and dynamics. Metadislocations were later on discovered in further CMAs, e.g. in T-Al-Mn- $-\mathrm{Pd}^{71}$ and $\mathrm{Al}-\mathrm{Pd}-\mathrm{Fe}^{72}$ and seem to emerge as a general concept for the plastic deformation process in complex metal structures. ${ }^{73}$

\section{Acknowledgements}

I thank all students, many of which later became colleagues, who have worked with me on this topic over the years. Results of Volker Franz, Marc Heggen, Thomas Lange, Christoph Metzmacher, Ronald Rosenfeld, and Peter Schall were incorporated in this review. The Post-Docs and guests in my group,
Nobumichi Tamura, Wenge Yang, and Renhui Wang greatly contributed with their ideas and knowledge. Finally I gratefully acknowledge the work of M. Beyss, M. Schmidt, and C. Thomas who successfully developed growth routes for many quasicrystal phases and carried out all the necessary preliminaries.

\section{References}

1 J. Kepler, The Harmony of the World, American Philosophical Society, Philadelphia, 1997.

2 D. Shechtman, I. Blech, D. Gratias and J. W. Cahn, Phys. Rev. Lett., 1984, 53, 1951.

3 D. Levine, T. C. Lubensky, S. Ostlund, S. Ramaswamy and P. J. Steinhard, Phys. Rev. Lett., 1985, 54, 1520.

4 K. Hiraga and M. Hirabayashi, Jpn. J. Appl. Phys., 1987, 26, L155.

5 D. N. Wang, T. Ishimasa, H. U. Nissen and S. Hovmöller, Mater. Sci. Forum, 1987, 22-24, 381.

6 Z. Zhang and K. Urban, Philos. Mag. Lett., 1989, 60, 97.

7 P. Schall, M. Feuerbacher and K. Urban, Phys. Rev. [Sect.] B, 2004, 69, 134105.

8 P. Schall, M. Feuerbacher and K. Urban, Philos. Mag. Lett., 2004, 84, 471.

9 J. E. S. Socolar, T. C. Lubensky and P. J. Steinhard, Phys. Rev. [Sect.] B, 1986, 34, 3345.

10 M. Duneau and A. Katz, Phys. Rev. Lett., 1985, 54, 2688.

11 A. Katz and M. Duneau, J. Phys., Colloq., 1986, 47(C3), 103.

12 J. W. Cahn, D. Shechtman and D. Gratias, J. Mater. Res., 1986, $1,13$.

13 C. Janot, Quasicrystals - a primer, Clarendon Press, Oxford, 2nd edn, 1996.

14 M. Boudard, M. de Boissieu, C. Janot, G. Heger, C. Beeli, H.-U. Nissen, H. Vincent, R. Ibbertson, M. Audier and J. M. Dubois, J. Phys.: Condens. Matter, 1992, 4, 10149.

15 M. Cornier-Quiquandon, A. Quivy, S. Lefebvre, E. Elkaim, G. Heger, A. Katz and D. Gratias, Phys. Rev. [Sect.] B, 1991, 44, 2071.

16 A. Katz and D. Gratias, in Lectures on Quasicrystals, ed. F. Hippert and D. Gratias, Les Éditions de Physique, Les Ulis, 1994, p. 187.

$17 \mathrm{~J}$. Bohsung and H.-R. Trebin, in Aperiodicity and Order, ed. M. J. Jarić, Academic Press, New York, 1989, p. 183.

18 M. Kléman and Ch. Sommers, Acta Metall. Mater., 1991, 39, 287.

19 W. Yang, M. Feuerbacher, N. Tamura, D. Ding, R. Wang and K. Urban, Philos. Mag. A, 1998, 77, 1481.

20 M. Feuerbacher, C. Metzmacher, M. Wollgarten, B. Baufeld, M. Bartsch, U. Messerschmidt and K. Urban, Mater. Sci. Eng. A, 1997, 233(1-2), 103.

21 K. Edagawa and S. Takeuchi, in Dislocations in Solids, ed. F. R. N. Nabarro and J. P. Hirth, Elsevier, Den Haag, 2007, vol. 13, p. 365.

22 D. H. Ding, W. Yang, C. Hu and R. Wang, Mater. Sci. Forum, 1994, 150-151, 345.

23 D. H. Ding, R. Wang, W. Yang and C. Hu, J. Phys.: Condens. Matter, 1995, 7, 5423.

24 M. Kléman, Czech. J. Phys., 1995, 45, 935.

25 D. Gratias, J. T. Beauchesne, F. Mompiou and D. Caillard, Philos. Mag., 2006, 86, 4139.

26 D. B. Williams and C. B. Carter, Transmission electron microscopy A textbook for materials science, Springer Press, New York, 2nd edn, 2009.

27 P. Hirsch, A. Howie, R. Nicholson, D. W. Pashley and M. J. Whelan, Electron microscopy of thin crystals, Krieger publishing, Malabar, 2nd edn, 1977.

28 M. Wollgarten, D. Gratias, Z. Zhang and K. Urban, Philos. Mag. A, 1991, 64, 819.

29 F. Mompiou, L. Bresson, P. Cordier and D. Caillard, Philos. Mag. A, $2003, \mathbf{8 3}, 3133$.

30 D. Cherns and A. R. Preston, Proc. XIth Int. Congr. on Electr. Micr., 1986, vol. 1, p. 721.

31 R. Wang and M. X. Dai, Philos. Mag. Lett., 1990, 61, 119.

32 W. Yang, R. Wang, M. Feuerbacher, P. Schall and K. Urban, Philos. Mag. Lett., 2000, 80, 281.

33 J. Devaud-Rzepski, M. Cornier-Quinquandon and D. Gratias, in Quasicrystals and Incommensurate Structures in Condensed Matter, 
ed. M. J. Yacaman, D. Romen, V. Castano and A. Gomez, World Scientific, Singapore, 1990, p. 498.

34 J. Bonneville, D. Caillard and P. Guyot, in Dislocations in Solids, ed. F. R. N. Nabarro and J. P. Hirth, Elsevier, Den Haag, 2008, vol. 14, p. 253.

35 R. Rosenfeld, M. Feuerbacher, B. Baufeld, M. Bartsch, U. Messerschmidt, M. Wollgarten, G. Hanke, M. Beyss and K. Urban, Philos. Mag. Lett., 1995, 72, 375.

36 M. Feuerbacher, C. Metzmacher, M. Wollgarten, K. Urban, B. Baufeld, M. Bartsch and U. Messerschmidt, Mater. Sci. Eng., A, 1997, 226-228, 943.

37 F. Mompiou, D. Caillard and M. Feuerbacher, Philos. Mag., 2004, 84, 2777.

38 F. Mompiou, L. Bresson, P. Cordier and D. Caillard, Philos. Mag., 2003, 83, 3133.

39 M. X. Dai, Philos. Mag. A, 1993, 67, 789.

40 J. Feng, R. Wang and Z. Wang, Philos. Mag. Lett., 1993, 68, 321.

41 R. Wang, M. Feuerbacher, M. Wollgarten and K. Urban, Philos. Mag. A, 1998, 77, 523.

42 M. Wollgarten, C. Metzmacher, R. Rosenfeld and M. Feuerbacher, Philos. Mag. A, 1997, 76, 445.

43 M. X. Dai, Philos. Mag. Lett., 1992, 66, 235.

44 R. Wang, X. Yang, W. Zou, Z. Wang, J. Gui and J. Jiang, J. Phys.: Condens. Matter, 1994, 6, 9009.

45 M. Wollgarten and K. Urban, Philos. Mag. Lett., 1993, 68, 1993.

46 J. Feng and R. Wang, Philos. Mag. Lett., 1994, 69, 309.

47 C. Metzmacher, Diploma Thesis RWTH Aachen, 1995.

48 R. Wang, M. Feuerbacher, W. Yang and K. Urban, Philos. Mag. A, 1998, 78, 273.

49 D. Caillard, G. Vanderschaeve, L. Bresson and D. Gratias, Philos. Mag. A, 2000, 80, 237.

50 M. Texier, J. Bonneville, A. Proult, L. Thilly and J. Rabier, Mater. Sci. Eng., A, 2005, 400-401, 311.

51 U. Messerschmidt, M. Bartsch, B. Geyer, L. Ledig, M. Feuerbacher, M. Wollgarten and K. Urban, Mater. Res. Soc. Symp. Proc., 2001, 643, K6.5.1.

52 S. Takeuchi, R. Tamura, E. Kabutoya and K. Edagawa, Philos. Mag. A, 2002, 82, 379.
53 U. Messerschmidt, D. Häussler, M. Bartsch, B. Geyer, M. Feuerbacher and K. Urban, Mater. Sci. Eng., A, 2000, 294, 757.

54 M. Feuerbacher, P. Schall, Y. Estrin and Y. Bréchet, Philos. Mag. Lett., 2001, 81, 473.

55 P. Schall, M. Feuerbacher, U. Messerschmidt and K. Urban, Philos. Mag. Lett., 1999, 79, 785.

56 P. A. Bancel and P. A. Heiney, J. Physiol. (Paris), 1986, C3, 341.

57 D. N. Wang, T. Ishimasa, H. U. Nissen, S. Hovmöller and J. Rhyner, Philos. Mag. A, 1988, 58, 737.

58 Y. Ishii, Phys. Rev. [Sect.] B, 1989, 39, 862.

59 M. Feuerbacher and D. Caillard, Acta Mater., 2006, 54, 3233.

60 R. Mikulla, J. Roth and H.-R. Trebin, Philos. Mag. B, 1995, 71, 981.

61 Z. Luo, S. Zhang, Y. Tang and D. Zhao, Scr. Metall., 1993, 28, 1513 .

62 M. Heggen, M. Feuerbacher, P. Schall, K. Urban and R. Wang, Phys. Rev. [Sect.] B, 2001, 64, 14202.

63 T. Ohno and T. Ishimasa, Proc. ICQ6, World Scientific, Singapore, 1998, p. 39.

64 T. Ishimasa, K. Oyamada, Y. Arichika, E. Nishibori, M. Takata, M. Sakata and K. Kato, J. Non-Cryst. Solids, 2004, 334-335, 167.

65 J. Devaud-Rzepski, A. Quivy, Y. Calvayrac, M. Cornier-Quiquandon and D. Gratias, Philos. Mag. B, 1989, 60, 855.

66 M. Heggen, M. Feuerbacher, T. Lange and K. Urban, J. Alloys Compd., 2002, 342, 330.

67 H. Takakura, C. Pay Gómez, A. Yamamoto, M. De Boissieu and A. P. Tsai, Nat. Mater., 2007, 6, 58.

68 A. P. Tsai, T. Masumoto and A. Yamamoto, Philos. Mag. Lett., 1992, 66, 203

69 K. Urban and M. Feuerbacher, J. Non-Cryst. Solids, 2004, 334-335, 143.

70 H. Klein, M. Feuerbacher, P. Schall and K. Urban, Phys. Rev. Lett., 1999, 82, 3468.

71 M. Heggen, L. Houben and M. Feuerbacher, Nat. Mater., 2010, 9, 332 .

72 M. Feuerbacher, S. Balanetskyy and M. Heggen, Acta Mater., 2008, 56, 1849.

$73 \mathrm{M}$. Feuerbacher and M. Heggen, in Dislocations in Solids, ed. J. P. Hirth and L. Kubin, Elsevier, Den Haag, 2009, vol 16, p. 110. 